\title{
A Rank-Reduced LMMSE Canceller for Narrowband Interference Suppression in OFDM-Based Systems
}

\author{
Rickard Nilsson, Associate Member, IEEE, Frank Sjöberg, Member, IEEE, and James P. LeBlanc, Senior Member, IEEE
}

\begin{abstract}
We present a narrowband interference (NBI) canceller that suppresses spectral leakage in an orthogonal frequency-division multiplexing (OFDM)-based system caused by a narrowband (NB) signal. In our scenario, we assume that the spectrum of the NB signal is within the spectrum of the OFDM signal. This can be the case, e.g., on digital subscriber lines (DSL) and in new unlicensed frequency bands for radio transmission.

The canceller makes linear minimum mean-square error estimates of the spectral leakage by measuring the NBI on a few modulated or unmodulated OFDM subcarriers. It uses a model of the NB signal's power spectral density as a priori information. Using frequency invariant design it is possible to cancel NBI from signals that are changing their frequency location with significantly reduced complexity overhead. The operational complexity of the canceller can be lowered by using the theory of optimal rank reduction and using the time-bandwidth product of the NB signal.

Analytical performance evaluations, as well as Monte Carlo simulations, also show that without perfect $a$ priori information this canceller can suppress the spectral leakage from a strong NB signal (e.g., with equal power as the OFDM signal) to well below the background noise floor for typical applications where it causes negligible signal to noise ratio and symbol error rate degradation.
\end{abstract}

Index Terms-Digital subscriber lines (DSL), discrete multitone (DMT), industrial-scientific-medical (ISM) band, orthogonal frequency-division multiplexing (OFDM), radio frequency interference (RFI), wireless local area network (WLAN).

\section{INTRODUCTION}

I N THIS PAPER, we present a new narrowband interference (NBI) canceller that suppresses the spectral leakage from an inband narrowband (NB) signal in orthogonal frequency-division multiplexing (OFDM)-based systems. This type of signal interference may arise in many new communication systems as the spectrum becomes more crowded. Examples of NBI in wireline systems are radio frequency interference (RFI) from short-wave radio, Citizen's Band (CB) radio, and amateur radio (HAM), which interferes with hybrid fiber coaxial (HFC) networks [1] and digital subscriber lines (DSL) [2]. Ineffective cable shielding of a network may also cause the ingress from external electrical devices in the homes, such as TVs, computers, etc. Similar scenarios of NBI may also be found in radio systems

Paper approved by M.-S. Alouini, the Editor for Modulation and Diversity Systems of the IEEE Communications Society. Manuscript received December 10, 2001; revised August 12, 2002, April 25, 2003, and May 15, 2003. This paper was presented in part at the European Personal Mobile Communications Conference, EPMCC01, Vienna, Austria, February 2001.

R. Nilsson is with the Telecommunications Research Center Vienna (ftw.), A-1220 Vienna, Austria (e-mail: nilsson@ftw.at).

F. Sjöberg and J. P. LeBlanc are with the Division of Signal Processing, Luleå University of Technology, SE-97187 Luleå, Sweden (e-mail: frank@sm.luth.se; leblanc@sm.luth.se).

Digital Object Identifier 10.1109/TCOMM.2003.820761 including the new unlicensed frequency bands, e.g., the industrial-scientific-medical (ISM) band, where an NB signal interferes with OFDM-based wireless local area networks (WLANs) such as Hiperlan II and IEEE 802.11a [3].

The general scenario in this paper is a strong NB signal, typically from another communication system, which resides within the same frequency band as a wideband OFDM signal. In this case, severe signal-to-noise ratio (SNR) degradation will occur across most OFDM subcarriers due to spectral leakage of the NB signal which is caused by the block processing in the OFDM receiver.

Some of the OFDM subcarriers are used by the canceller for measuring the NBI. Best performance is obtained if these subcarriers are silent (unmodulated), which is a requirement in certain system scenarios in order to achieve coexistence possibilities between the narrow- and the wideband systems. An example of this is multicarrier discrete multitione (DMT)-based DSL systems, which encounters inband RFI from amateur radio [2]. However, the canceller can also operate without using any silent measurement tones with a reduced, but still significant, performance improvement.

Very high bit-rate DSL (VDSL) is selected as a specific application scenario and we show how the canceller can be naturally incorporated in the receiver structure for a DMT-based VDSL system, standardized by the European Telecommunication Standards Institute (ETSI) [4], and show performance examples of cancelling inband RFI caused by HAM. However, the same receiver structure can also be used for OFDM-based radio application systems. As such, we show how to cancel strong inband NBI in an OFDM system without using any silent measurement tones and by using a standard block code to correct errors caused by the residual NBI after cancellation.

The canceller makes linear minimum mean-square error (LMMSE) estimates of the NBI at the receiver by measuring the interference on a few of the subcarriers close to the NB signal's center frequency. By its construction, the canceller can be designed to suppress interference from NB signals that change their carrier frequency, without updating the estimator coefficients. This reduces the complexity overhead considerably. The canceller needs a priori information about the NB signal's power spectral density (PSD) in its design. However, the NB signal's PSD is generally unknown, but robust suppression results can still be obtained with only a rough knowledge of the bandwidth and center-frequency location of the NB signal. Further, we achieve a low-complexity approximation of the LMMSE estimator by applying the theory of optimal rank reduction [5] and the time-bandwidth product of the NB signal [6]. 


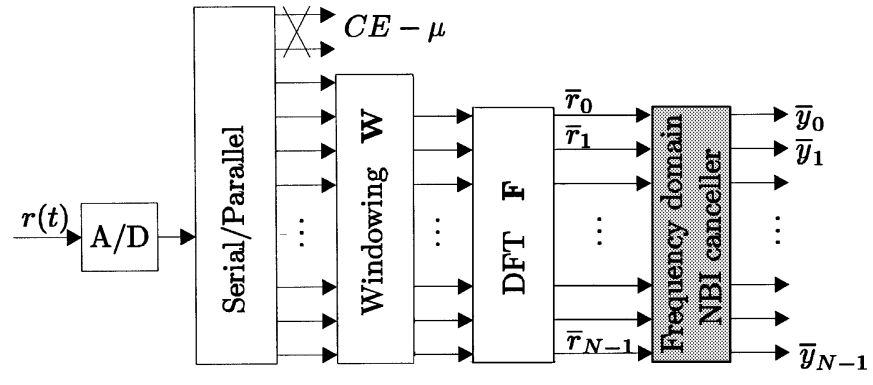

Fig. 1. Baseband OFDM receiver with the frequency-domain NBI canceller shaded.

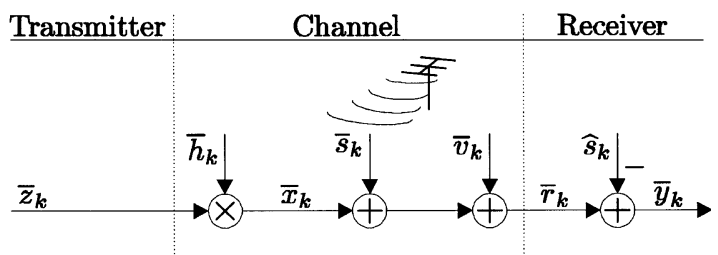

Fig. 2. Signal path at one OFDM subchannel with spectral leakage, $\bar{s}_{k}$, as interference from an NB signal.

With analytic and simulated performance evaluations we show that this canceller can suppress NBI in an OFDM system to below the background noise floor in typical application scenarios using silent tones. This is possible even if the total power of the NB signal is higher than for the OFDM signal and if the exact a priori information about the NB signal is unknown.

\section{SYSTEM MODEL}

In the system model, we assume a general OFDM system and an application scenario similar as DSL or an OFDM system that operates in unlicensed frequency bands for radio transmission, i.e., NB signals can overlap parts of the OFDM spectrum. In DSL, subcarriers at these overlapping frequencies must be silent (unmodulated) to obtain a cooperative environment with the NB system, due to the egress of modulated OFDM subcarriers which would interfere with the NB signal [7]. For radio applications that may not be the case in general. Although the canceller can cancel strong NBI quite successfully without silent measurement tones, the best performance is achieved if silent measurement tones are available.

Fig. 1 shows the baseband OFDM receiver used throughout this paper. We assume that the use of a cyclic extension (CE) preserves subcarrier orthogonality [8]. The number of subcarriers is $N$ and the length of the CE is $C E$. The CE can be extended with $\mu$ extra samples from what is required by the channel to preserve the subcarrier orthogonality. These $\mu$ extra samples, which are part of the CE, are used herein for a nonrectangular receiver windowing that maintains subcarrier orthogonality [9].

After windowing and discrete Fourier transform (DFT) processing we introduce a frequency-domain NBI canceller that measures the interference on a few (un)modulated subcarriers close to the NB signal's center frequency and subtracts NBI estimates from all subcarriers, see Fig. 2. This idea was introduced

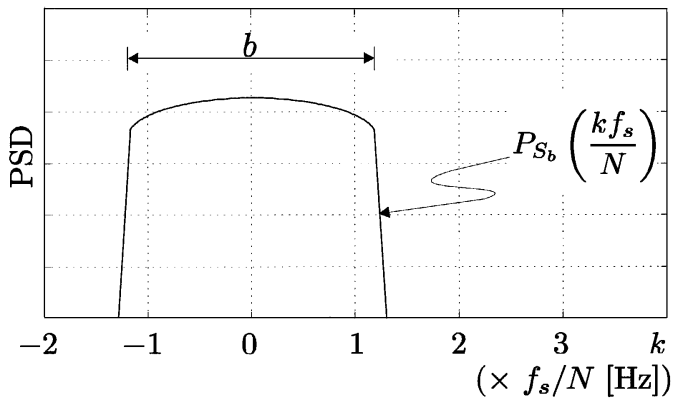

Fig. 3. Baseband PSD of NB signal with bandwidth $b \cdot f_{s} / N[\mathrm{~Hz}]$.

for DMT in [10], [11], by using unmodulated tones, for a deterministic sinusoid RF signal with known frequency. The same idea was applied in [12] and further expanded in [13] to handle RF signals with some unknown small bandwidth and a small uncertainty in its center-frequency location.

In this paper, we model the NB signal as a stochastic NB process with possibly unknown bandwidth and power spectrum. Furthermore, we assume that we have only a rough estimate of the center frequency of the NB signal. This canceller can be designed to be more robust than the cancellers mentioned above and it can suppress NB signals with wider bandwidth and larger uncertainty of its center-frequency location. Because MMSE estimators take the background noise into account, it can also be used without using silent measurement tones by modeling the received data on the measurement tones as part of the background noise.

\section{A. NB Signal Model}

The NB signal is modeled as an NB stochastic process and we model the received complex baseband signal as

$$
r(t)=x(t)+s(t)+v(t), \quad-\infty<t<\infty
$$

where $x(t)$ is the OFDM signal, $s(t)$ is an unknown NB signal, and $v(t)$ represents other background noise which we assume is additive white Gaussian noise (AWGN). The baseband NB signal is modeled as

$$
s(t)=s_{b}(t) e^{j 2 \pi f_{c} t}
$$

where $s_{b}(t)$ is a complex wide-sense stationary NB signal, centered around $f=0$. Its location relative to the baseband (down modulated) OFDM signal is represented by the center frequency $f_{c}$. We denote $s_{b}(t)$ 's PSD as $P_{S_{b}}(f)$ and the corresponding autocorrelation function as $r_{s_{b}}(\tau) \triangleq E\left\{s_{b}(t+\tau) s_{b}^{*}(t)\right\}$, where $E\{\cdot\}$ denotes expectation. Fig. 3 shows a sketch of $P_{S_{b}}(f)$ whose bandwidth is modeled to be $b \cdot f_{s} / N$, where $f_{s} / N$ is the OFDM subcarrier spacing, $f_{s}$ the sampling frequency of the received signal, and $b$ is the (possibly noninteger) number of subcarriers spanned.

For baseband systems like DSL, the NB signal is modeled as a corresponding real stochastic process

$$
s(t)=\Re\left\{s_{b}(t) e^{j 2 \pi f_{c} t}\right\} .
$$




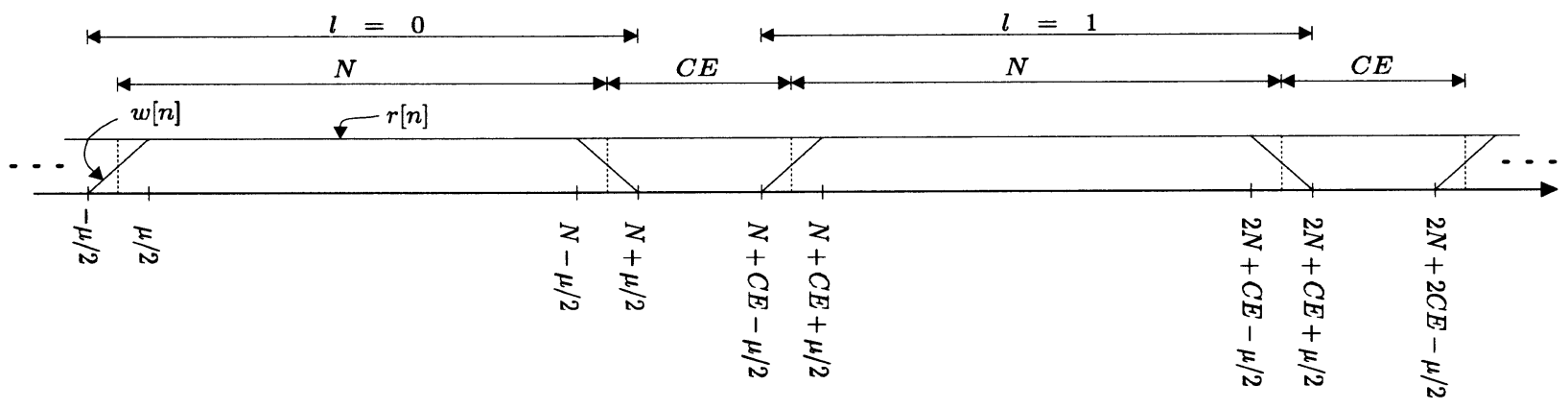

Fig. 4. Framing and windowing the OFDM signal $x[n]$ using a window function $w[n]$.

Note that this doubles the bandwidth of (2), for $f_{c}>b / 2$, since the frequency content of $s_{b}(t)$ is translated to both $f_{c}$ and $-f_{c}$. Furthermore, it is important to recognize that (2) is second-order circular, or proper, while the NB signal in (3) is not second-order circular, or improper [14], [15]. The latter will pose some special consequences for RFI cancellation in DMT-based DSL systems which will be discussed later.

These signal models account for many types of NB signals. The shape and bandwidth of $P_{S_{b}}(f)$ are design parameters for the LMMSE canceller derived in Section III. The worst case NB signal PSD for block processed signals is approximately flat [16], which is used in this paper as a design PSD when the true PSD of the NB signal is unknown.

\section{B. Receiver Framing and Windowing}

Let samples of the received signal be denoted as

$$
r[n]=r\left(\frac{n}{f_{s}}\right)=x[n]+s[n]+v[n] .
$$

Before windowing and DFT processing, the sampled signal is framed into blocks and the CE is removed. Let a long column vector of $N+\mu$ samples representing one OFDM frame be denoted as

$$
\begin{aligned}
r[l]=\left[r\left[l(N+C E)-\frac{\mu}{2}\right]\right. \\
\left.\cdots r\left[(l+1) N+l \cdot C E+\frac{\mu}{2}-1\right]\right]^{T} \\
=\mathbf{x}[l]+\mathbf{s}[l]+\mathbf{v}[l]
\end{aligned}
$$

where $l$ is a frame index with $l=0$ representing the current OFDM-frame being processed by the receiver. Note that the $\mu$ samples used for windowing are part of the $\mathrm{CE}$ samples (i.e., $\mu \leq C E$ ), see Fig. 4.

The framing (5) acts as a rectangular time-domain window on the NB signal which results in a spectral leakage to most OFDM subcarriers. Using a nonrectangular window $w[n]$ prior to the DFT processing, as sketched in Fig. 4, helps to reduce the spectral leakage on subcarriers further away from its center frequency by lowering the NBI sidelobes. Windowing can be used in combination with NBI cancellation and can reduce the number of subcarriers that need NBI cancellation, but reduces the efficiency due to an increased CE length.
Let the DFT of one windowed OFDM frame be expressed as

$$
\begin{aligned}
\underset{r}{\bar{r}[k]}= & \sum_{n=-\frac{\mu}{2}}^{N+\frac{\mu}{2}-1} w[n] r[n] e^{-j \frac{2 \pi n k}{N}} \\
= & \sum_{n=0}^{\frac{\mu}{2}-1}(w[n] r[n]+w[n+N] r[n+N]) e^{-j \frac{2 \pi n k}{N}} \\
& +\sum_{n=\frac{\mu}{2}}^{N-\frac{\mu}{2}-1} w[n] r[n] e^{-j \frac{2 \pi n k}{N}} \\
& +\sum_{n=N-\frac{\mu}{2}}^{N-1}(w[n] r[n]+w[n-N] r[n-N]) \\
& \times e^{-j \frac{2 \pi n k}{N}}
\end{aligned}
$$

From (8) it is seen that the $N$ point DFT taken over $N+\mu$ samples can be expressed as an $N$ point DFT taken over $N$ samples. Hence, independent of the window size $\mu$, the often fixed (by hardware) $N$-size fast Fourier transform (FFT) unit in an OFDM receiver can be maintained when using a nonrectangular window. Note also that the subcarrier orthogonality will be maintained when using a nonrectangular window if the $\mathrm{CE}$ is increased by $\mu$ samples $^{1}$ and if the window function is a pulse which fulfils the Nyquist criterion for intersymbol interference free signalling [9]. Then, the windowing will only have an impact on the noise parts of the received signal.

The windowing operation (8) can be interpreted as a frequency-domain decimation from $N+\mu$ down to $N$ samples, which leads to the time-domain folding, as sketched in Fig. 5. It can be represented with an $N \times(N+\mu)$ size matrix

$$
\begin{aligned}
& \mathbf{W}=\left[\begin{array}{ccccc}
\mathbf{0}_{\frac{\mu}{2}} & \mathbf{I}_{\frac{\mu}{2}} & \mathbf{0} & \mathbf{0}_{\frac{\mu}{2}} & \mathbf{I}_{\frac{\mu}{2}} \\
\mathbf{0} & \mathbf{0} & \mathbf{I}_{N-\mu} & \mathbf{0} & \mathbf{0} \\
\mathbf{I}_{\frac{\mu}{2}} & \mathbf{0}_{\frac{\mu}{2}} & \mathbf{0} & \mathbf{I}_{\frac{\mu}{2}} & \mathbf{0}_{\frac{\mu}{2}}
\end{array}\right] \\
& \times\left[\begin{array}{ccc}
w\left[-\frac{\mu}{2}\right] & 0 & 0 \\
0 & \ddots & 0 \\
0 & 0 & w\left[N+\frac{\mu}{2}-1\right]
\end{array}\right]
\end{aligned}
$$

where $\mathbf{0}_{x}$ is a square zero matrix and $\mathbf{I}_{x}$ is an identity matrix, both of size $x$. Then, a windowed OFDM frame can be expressed as $\mathbf{W r}[l]$.

$$
{ }^{1} \mu=0 \text { corresponds to a rectangular window. }
$$




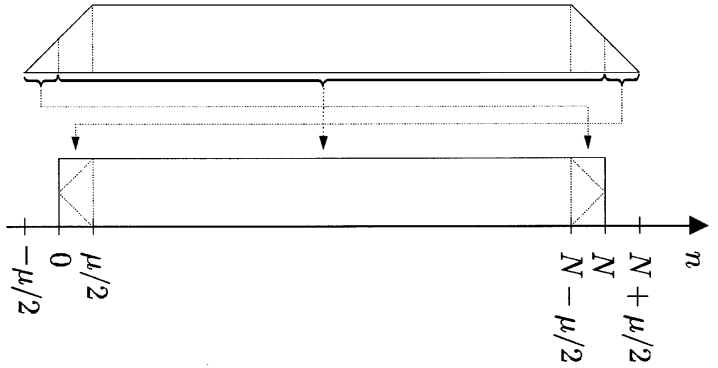

Fig. 5. Windowing operation.

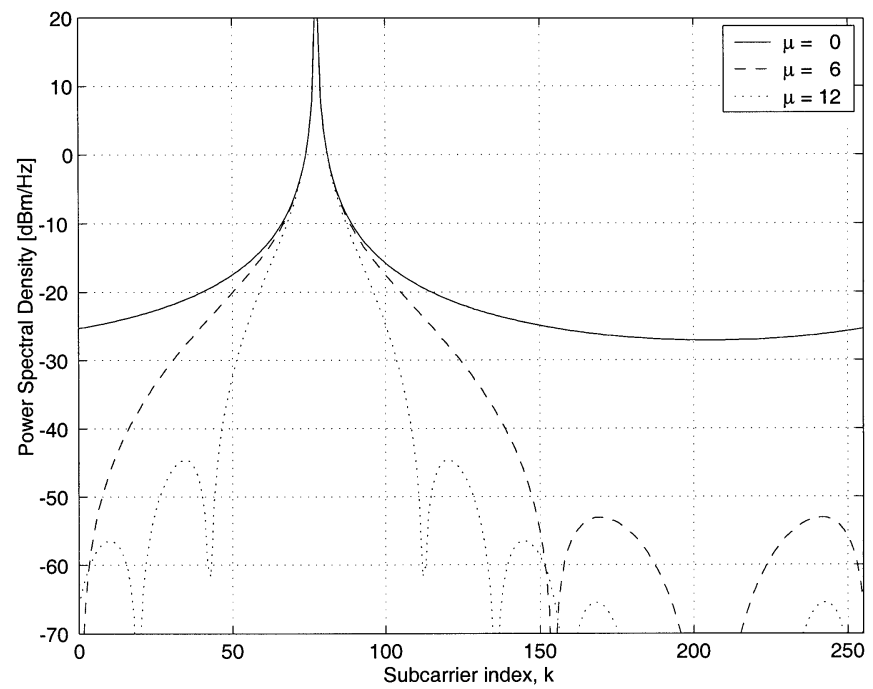

Fig. 6. PSD levels for the spectral leakage by a NB signal with bandwidth $b=1.0$. Raised cosine window using $\mu$ samples. $N=256$ subcarriers.

\section{DFT Processing}

After cyclic extension removal and windowing, the time domain OFDM frames are turned into the frequency domain by the DFT processing

$$
\begin{aligned}
\overline{\mathbf{r}}[l] & =\mathbf{F W r}[l]=\overline{\mathbf{x}}[l]+\overline{\mathbf{s}}[l]+\overline{\mathbf{v}}[l] \\
& =\left[\bar{r}_{0}[l] \cdots \bar{r}_{N-1}[l]\right]^{T}
\end{aligned}
$$

where the bar represents the equivalent frequency-domain signal and $\mathbf{F}$ is the orthonormal DFT matrix

$$
\mathbf{F}_{k, n}=\frac{1}{\sqrt{N}} e^{-j \frac{2 \pi k n}{N}}, \quad 0 \leq k, n \leq N-1
$$

Fig. 6 shows examples of the spectral leakage PSD from an NB signal with bandwidth $b=1.0\left(\times f_{s} / N[H z]\right)$ and its center frequency is located in between two OFDM subcarriers. A raised-cosine-shaped window was used with $\mu$ samples for windowing. Since the NB signal has a nonzero bandwidth the level of the spectral leakage onto neighboring subcarriers can vary greatly between different OFDM frames.

\section{LMMSE ESTIMATOR}

In this section, we derive an LMMSE canceller in the frequency domain of the OFDM signal, as shown in Fig. 1. It makes estimates of NB signal leakage onto all OFDM subcarriers by using only a few subcarriers located close to the center frequency of the NB signal as measurement tones.

The received signal on subcarrier $k$ is

$$
\bar{r}_{k}=\bar{x}_{k}+\bar{s}_{k}+\bar{v}_{k}, \quad 0 \leq k \leq N-1
$$

where $\bar{x}_{k}=\bar{z}_{k} \bar{h}_{k}$ is the OFDM signal part (with data $\bar{z}_{k}$, channel attenuation $\bar{h}_{k}$ ), $\bar{s}_{k}$ is the NBI leakage term, and $\bar{v}_{k}$ is AWGN. Estimates of the NBI leakage, $\widehat{s}_{k}$, are derived and then subtracted from each subcarrier

$$
\bar{y}_{k}=\bar{r}_{k}-\widehat{s}_{k}=\bar{x}_{k}+\left(\bar{s}_{k}-\widehat{s}_{k}\right)+\bar{v}_{k}, \quad 0 \leq k \leq N-1
$$

as shown in Fig. 2.

Since we assume that the interference signal $s(t)$ is NB relative to the OFDM signal the autocorrelation function will have support over several OFDM frames, that is, $r_{s}(\tau) \neq 0,|\tau|>$ $(N+C E) / f_{s}$. Thus, a somewhat improved performance can be achieved if NBI-measurements from a few sequential OFDM frames are used. We use this in the derivation of the estimator. Hence, let

$$
\mathbf{r}=\left[\mathbf{r}^{T}\left[-L_{n}\right] \cdots \mathbf{r}^{T}[0] \cdots \mathbf{r}^{T}\left[L_{p}\right]\right]^{T}
$$

represent an $\left(L_{p}+L_{n}+1\right)(N+\mu)$-long column vector with $L_{n}$ OFDM frames being processed prior and $L_{p}$ frames being processed after frame 0 , which is the current frame we consider for NBI cancellation.

Let $P=L_{p}+L_{n}+1$ denote the number of frames used for NBI measurements. Using $L_{p}>0$ introduces an extra delay in the receiver by $L_{p}(N+C E) / f_{s}$ seconds. By using the weak stationary assumption (2), the correlation matrix for the NBI component of the received signal becomes

$$
\begin{aligned}
& \mathbf{R}_{\mathbf{s}}=E\left\{\mathbf{s s}^{H}\right\} \\
& =\left[\begin{array}{ccc}
E\left\{\mathbf{s}\left[-L_{n}\right] \mathbf{s}^{H}\left[-L_{n}\right]\right\} & \cdots & E\left\{\mathbf{s}\left[-L_{n}\right] \mathbf{s}^{H}\left[L_{p}\right]\right\} \\
\vdots & \ddots & \vdots \\
E\left\{\mathbf{s}\left[L_{p}\right] \mathbf{s}^{H}\left[-L_{n}\right]\right\} & \cdots & E\left\{\mathbf{s}\left[L_{p}\right] \mathbf{s}^{H}\left[L_{p}\right]\right\}
\end{array}\right] \\
& =\left[\begin{array}{ccc}
\mathbf{R}_{\mathbf{s}}[0] & \cdots & \mathbf{R}_{\mathbf{s}}\left[-L_{n}-L_{p}\right] \\
\vdots & \ddots & \vdots \\
\mathbf{R}_{\mathbf{s}}\left[L_{p}+L_{n}\right] & \cdots & \mathbf{R}_{\mathbf{s}}[0]
\end{array}\right]
\end{aligned}
$$

with $\mathbf{R}_{\mathbf{s}}[k]=\mathbf{R}_{\mathbf{s}}^{H}[-k]$ symmetry, where $(\cdot)^{H}$ denotes the Hermitian transpose. The frequency-domain equivalent of $\mathbf{R}_{\mathbf{s}}$ is

$$
\begin{aligned}
\mathbf{R}_{\overline{\mathbf{s}}} & =E\left\{\overline{\mathbf{s s}}^{H}\right\} \\
& =\left[\begin{array}{ccc}
\mathbf{R}_{\overline{\mathbf{s}}}[0] & \cdots & \mathbf{R}_{\overline{\mathbf{s}}[}\left[-L_{n}-L_{p}\right] \\
\vdots & \ddots & \vdots \\
\mathbf{R}_{\overline{\mathbf{s}}}\left[L_{p}+L_{n}\right] & \cdots & \mathbf{R}_{\overline{\mathbf{s}}[0]}[0]
\end{array}\right]
\end{aligned}
$$

where

$$
\mathbf{R}_{\overline{\mathbf{s}}}[k]=\mathbf{F W R}_{\mathbf{s}}[k] \mathbf{W}^{H} \mathbf{F}^{H}=\mathbf{R}_{\overline{\mathbf{s}}}^{H}[-k] .
$$

As discussed earlier, assume for now that the subcarriers located very close to the peak interference are unused. Hence, let 
$\bar{x}_{k}=0, k \in \omega$ where $\omega$ represents the index set of the silent tones used for measuring the NBI and let

$\bar{r}_{k}[l]=\bar{s}_{k}[l]+\bar{v}_{k}[l],\left\{\begin{array}{l}k \in \omega=\left\{m_{1}, \ldots, m_{M}\right\} \\ l \in\left\{-L_{n}, \ldots, L_{p}\right\}, \quad-L_{n} \leq 0 \leq L_{p}\end{array}\right.$

be the measurements of the NBI leakage on these tones.

Let the $M$ measurements from each of the $P=$ $L_{p}+L_{n}+1$ frames be collected in vectors $\bar{r}_{\omega}[l]=$ $\left[\bar{r}_{m_{1}}[l] \ldots \bar{r}_{m_{M}}[l]\right]^{T}$ and stacked into one column vector $\overline{\mathbf{r}}_{\omega}=\left[\overline{\mathbf{r}}_{\omega}\left[-L_{n}\right]^{T} \ldots \overline{\mathbf{r}}_{\omega}\left[L_{p}\right]^{T}\right]^{T}$. Further, let the NBI in the current frame be represented by the vector $\overline{\mathbf{s}}[0]=\left[\bar{s}_{0}[0] \cdots \bar{s}_{N-1}[0]\right]^{T}$. We construct an LMMSE estimate [5] of $\overline{\mathbf{s}}[0]$ as

$$
\widehat{\mathbf{s}}[0]=\mathbf{K} \overline{\mathbf{r}}_{\omega}=\mathbf{R}_{\overline{\mathbf{s}}[0] \overline{\mathbf{r}}_{\omega}} \mathbf{R}_{\overline{\mathbf{r}}_{\omega} \overline{\mathbf{r}}_{\omega}}^{-1} \overline{\mathbf{r}}_{\omega}
$$

where

$$
\begin{aligned}
& \mathbf{R}_{\overline{\mathbf{s}}[0] \overline{\mathbf{r}}_{\omega}}=E\left\{\overline{\mathbf{s}}[0] \overline{\mathbf{r}}_{\omega}^{H}\right\}=E\left\{\overline{\mathbf{s}}[0] \overline{\mathbf{s}}_{\omega}^{H}\right\}=\mathbf{A} \mathbf{R}_{\overline{\mathbf{s}}} \mathbf{B} \\
& \mathbf{R}_{\overline{\mathbf{r}}_{\omega} \overline{\mathbf{r}}_{\omega}}=E\left\{\overline{\mathbf{r}}_{\omega} \overline{\mathbf{r}}_{\omega}^{H}\right\}=E\left\{\overline{\mathbf{s}}_{\omega} \overline{\mathbf{s}}_{\omega}^{H}\right\}+\sigma^{2} \mathbf{I} \\
& =\mathbf{B}^{T} \mathbf{R}_{\overline{\mathbf{S}}} \mathbf{B}+\sigma^{2} \mathbf{I} \\
& \mathbf{A}=\left[\begin{array}{lll}
\mathbf{0}_{N \times N \cdot L_{n}} & \mathbf{I}_{N \times N} & \mathbf{0}_{N \times N \cdot L_{p}}
\end{array}\right]_{N \times P \cdot N} \\
& \mathbf{B}=\left[\begin{array}{ccc}
\mathbf{C}_{N \times M} & \mathbf{0} & \mathbf{0} \\
\mathbf{0} & \ddots & \mathbf{0} \\
\mathbf{0} & \mathbf{0} & \mathbf{C}_{N \times M}
\end{array}\right]_{P \cdot N \times P \cdot M}
\end{aligned}
$$

where $\sigma^{2}$ is the variance of the AWGN $\bar{v}_{k}$, and $\mathbf{A}, \mathbf{B}$ and $\mathbf{C}$ are indicator matrices. The size $-(N \times M)$ matrix $\mathbf{C}$ is all zeros except for $M$ ones positioned on different rows and columns representing how the measurement tones $\omega=\left\{m_{1}, \ldots, m_{M}\right\}$ are located in $[0, \ldots, N-1]$. That is, if $m_{i}$ is a measurement tone, then $\mathbf{C}_{m_{i}, i}=1$ otherwise $\mathbf{C}_{m_{i}, i}=0$.

Note that for the improper baseband NBI case (2), the widely linear MMSE estimator of $\overline{\mathbf{s}}[0]$ [15], [17] is easily obtained by also taking into account for the complex symmetric pairs of the selected measurement tones, $\bar{r}_{N-k}=\bar{r}_{k}^{*}$, in the formulation of the estimator (21). Then, since (2) is real, $\mathbf{R}_{\overline{\mathbf{s}}}$ automatically becomes the augmented covariance matrix which contains the complementary (or pseudo) covariance matrix structures, with elements $E\left\{\bar{s}_{m} \bar{s}_{n}\right\}$, besides the normal covariance matrix with elements $E\left\{\bar{s}_{m} \bar{s}_{n}^{*}\right\}$. Hence, all the second-order information about $\overline{\mathbf{s}}$ will then be contained in a widely linear estimator $\mathbf{K}$.
When no silent measurement tones are used, (20) will be in the form $\bar{r}_{k}[l]=\bar{x}_{k}[l]+\bar{s}_{k}[l]+\bar{v}_{k}[l]$, where $\bar{x}_{k}[l]$ is the data on subcarrier $k$ in OFDM frame $l$. By assuming that the transmitted data is independent and unknown at the receiver the autocorrelation of the measurement tones in (23) changes to

$$
\begin{aligned}
\mathbf{R}_{\overline{\mathbf{r}}_{\omega} \overline{\mathbf{r}}_{\omega}} & =E\left\{\overline{\mathbf{x}}_{\omega} \overline{\mathbf{x}}_{\omega}^{H}\right\}+E\left\{\overline{\mathbf{s}}_{\omega} \overline{\mathbf{s}}_{\omega}^{H}\right\}+\sigma^{2} \mathbf{I} \\
& =\mathbf{B}^{T} \mathbf{R}_{\overline{\mathbf{s}}} \mathbf{B}+\left(\sigma_{x}^{2}+\sigma^{2}\right) \mathbf{I}
\end{aligned}
$$

where $\sigma_{x}^{2}$ is the average received data power. Without loss of generality it is assumed to be equal on each subcarrier. Furthermore, it is of course also possible to have a mixture of silent and nonsilent measurement tones where the autocorrelation matrix then follows from (23) and (25).

The elements in the correlation matrix (18), which build up $\mathbf{K}$, are of the form shown by (26)-(28) at the bottom of the page where $P_{S_{b}}(f)$ and $f_{c}$ are a priori information about $s(t)$ for this LMMSE estimator, see Fig. 3. As will be demonstrated in Section IV it suffices to assume a flat NB model of $s(t)$ with a bandwidth $b$ that is at least as wide as the bandwidth of the true NB signal and centered around $f_{c}$ to obtain a robust canceller. That will effectively suppress NB signals that have a bandwidth less than or equal to $b \cdot f_{s} / N$. A simple estimate of $f_{c}$ can be obtained by using the squared magnitude of the FFT outputs, as in a periodogram, searching for the subcarriers with the strongest interference and interpolate between those for the location of $f_{c}$ [11], [13].

\section{A. Frequency Invariance}

If $P_{S_{b}}(f)$ needs to be changed a new correlation matrix needs to be recomputed, as in (19). However, it is possible to design the canceller so that its estimator coefficients remain the same if only the center frequency, $f_{c}$, of the NB signal has changed, to get exactly the same performance without performing a complex recomputation of the estimator coefficients. The theoretical conditions for this are as follows.

1) The frequency shift is an integer multiple of the subcarrier spacing.

Remark 1: In practice, by using a robust canceller design, frequency shifts that are noninteger multiples of the subcarrier spacing can also be handled, see Section IV.

2) The measurement tones are shifted accordingly to the new frequency location.

3) Only measurement tones in the current frame are used $\left(L_{p}=L_{n}=0\right)$.

$$
\begin{aligned}
E\left\{\bar{s}_{k}[p] \bar{s}_{l}^{*}[q]\right\} & =\frac{1}{N} \sum_{n=0}^{N-1} \sum_{m=0}^{N-1} E\left\{s\left(\frac{n+p(N+C E)}{f_{s}}\right) s^{*}\left(\frac{m+q(N+C E)}{f_{s}}\right)\right\} \times e^{-j \frac{2 \pi[n k-m l]}{n}} \\
& =\frac{1}{N} \sum_{n=0}^{N-1} \sum_{m=0}^{N-1} r_{s_{b}}\left(\frac{n-m+(p-q)(N+C E)}{f_{s}}\right) \times e^{j \frac{2 \pi f_{c}(n-m+(p-q)(N+C E))}{f_{s}}} e^{-j \frac{2 \pi[n k-m l]}{N}} \\
& =\frac{1}{N} \sum_{n=0}^{N-1} \sum_{m=0}^{N-1}\left(\int_{-\infty}^{\infty} P_{s_{b}}\left(f-f_{c}\right) e^{j \frac{2 \pi f[n-m+(p-q)(N+C E)]}{f_{s}}} d f\right) \times e^{-j \frac{2 \pi[n k-m l]}{N}}
\end{aligned}
$$


4) A proper NB signal of the analytic type (2) is considered.

Remark 2: For improper NB signals (3) this can also be handled in practice by using nonrectangular receiver windowing that sufficiently suppresses the correlation contribution between the positive and negative frequency peaks (located at $\pm f_{c}$ ).

The estimator (21) has an inherent invariance to frequency shifts due to the properties of the discrete Fourier transform (DFT). This will be demonstrated in the following. If the center frequency of the NB signal (2) shifts an integer multiples of subcarriers

$$
f_{c_{2}}=f_{c_{1}}+\alpha\left(\frac{f_{s}}{N}\right), \quad \alpha \in Z
$$

and given that the NBI is proper as in (2), it can be shown that the correlation matrix (18) becomes block rotated

$$
\widetilde{\mathbf{R}}_{\overline{\mathbf{s}}}=\left[\begin{array}{ccc}
\widetilde{\mathbf{R}}_{\overline{\mathbf{s}}}[0] & \cdots & \widetilde{\mathbf{R}}_{\overline{\mathbf{s}}}\left[-L_{n}-L_{p}\right] \\
\vdots & \ddots & \vdots \\
\widetilde{\mathbf{R}}_{\overline{\mathbf{s}}}\left[L_{p}+L_{n}\right] & \cdots & \widetilde{\mathbf{R}}_{\overline{\mathbf{s}}}[0]
\end{array}\right]
$$

where the $(k, l)$ th element, $E\left\{\bar{s}_{k}[m+n] \bar{s}_{l}^{*}[m]\right\}$, in each block matrix is

$$
\begin{gathered}
\widetilde{\mathbf{R}}_{\overline{\mathbf{s}}}[n]_{(k, l)}=\mathbf{R}_{\overline{\mathbf{s}}}[n]_{((k+\alpha) \bmod N,(l+\alpha) \bmod N)}, \\
0 \leq k, \quad l \leq N-1
\end{gathered}
$$

where mod represents the modulo operator. When a frequency shift occurs, as in (29), the measurement tones $\omega$ should be rotated accordingly

$$
\widetilde{\omega}=\{(k+\alpha) \bmod N \mid k \in \omega\}
$$

to reflect the new frequency location of the NB signal.

For $L_{p}=L_{n}=0$, i.e., when only measurement tones in the present OFDM-frame are used, $\widetilde{\mathbf{R}}_{\overline{\mathbf{s}}}=\widetilde{\mathbf{R}}_{\overline{\mathbf{s}}}[0]$. If the measurement tones are updated as in (32), the matrix $\mathbf{R}_{\overline{\mathbf{r}}_{\omega} \overline{\mathbf{r}}_{\omega}}$ remain the same, $\widetilde{\mathbf{R}}_{\overline{\mathbf{r}}_{\omega} \overline{\mathbf{r}}_{\omega}}=\mathbf{R}_{\overline{\mathbf{r}}_{\omega} \overline{\mathbf{r}}_{\omega}}$ and the rows are rotated for $\widetilde{\mathbf{R}}_{\overline{\mathbf{s}}[0] \overline{\mathbf{r}}_{\omega}(k, l)}=\mathbf{R}_{\overline{\mathbf{s}}_{[0]} \overline{\mathbf{r}}_{\omega}((k+\alpha) \bmod N, l)}$. This results in that the estimator-coefficients $\mathbf{K}$ in (21) only become rotated in the rows

$\widetilde{\mathbf{K}}_{(k, l)}=\mathbf{K}_{((k+\alpha) \bmod N, l)}, \quad 0 \leq k \leq N-1 ; \quad 0 \leq l \leq M-1$.

Alternatively, let the estimator coefficients remain unchanged, $\widetilde{\mathbf{K}}_{(k, l)}=\mathbf{K}_{(k, l)}$, and perform instead a rotation of the NBI estimates before they are cancelled from the subcarriers

$$
\widetilde{\widehat{\mathbf{s}}}[0]=\widehat{\mathbf{s}}[0]_{((k+\alpha) \bmod N, l),}, \quad 0 \leq k \leq N-1 .
$$

Consequently, if a frequency shift of the NB signal is suddenly detected, e.g., by monitoring the FFT outputs as a periodogram to locate the NBI peak, the measurement tones should only be shifted to around the new NBI-peak location, as they were at the previous frequency location, and the estimated interference rotated into position as in (34). No other changes need to be performed in the cancellation procedure. As a result, it becomes easier and saves a lot of computational complexity to cancel a NB signal which may change its frequency location, or to independently cancel spectral leakage from several NB signals in different frequency bands.

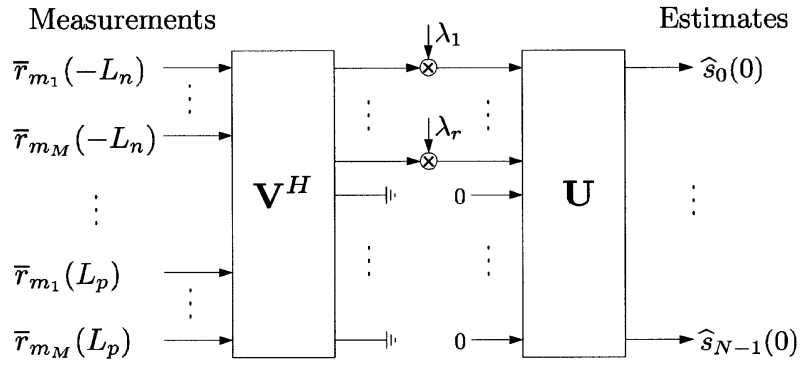

Fig. 7. Low-rank estimator. $M$ tones in $\left(L_{p}+L_{n}+1\right)$ frames are used to estimate interference in one frame ( $N$ subcarriers).

\section{B. Optimal Low-Rank Approximation}

To lower the computational complexity the Wiener estimator $\widehat{\mathbf{s}}[0]=\mathbf{K} \overline{\mathbf{r}}_{\omega}$ in (21) can be simplified with a low-rank approximation $\widehat{\mathbf{s}}_{r}[0]=\mathbf{K}_{r} \overline{\mathbf{r}}_{\omega}$ using singular value decomposition (SVD). This is similar to the work in [18] where a Wiener estimator for OFDM-channel estimation was rank reduced using SVD to lower the complexity. For this estimator the optimal low-rank approximation of $\mathbf{K}$ is [5]

$$
\mathbf{K}_{\mathbf{r}}=\mathbf{U} \boldsymbol{\Sigma}_{r} \mathbf{Q}^{H} \mathbf{R}_{\overline{\mathbf{r}}_{\omega} \overline{\mathbf{r}}_{\omega}}^{-\frac{1}{2}}=\mathbf{U} \boldsymbol{\Sigma}_{r} \mathbf{V}^{H}
$$

where $\mathbf{U}$ and $\mathbf{Q}$ are unitary matrices from the SVD of $\mathbf{K} \mathbf{R}_{\overline{\mathbf{r}}_{\omega} \overline{\mathbf{r}}_{\omega}}^{1 / 2}=\mathbf{U} \boldsymbol{\Sigma} \mathbf{Q}^{H}$ and where $\boldsymbol{\Sigma}_{r}$ is a size $N \times M P$ matrix containing the $r$ most significant singular values (out of $M P$ ), $\lambda_{1} \geq \cdots \geq \lambda_{r}$, from $\boldsymbol{\Sigma}$ along its diagonal

$$
\boldsymbol{\Sigma}_{r}=\operatorname{diag}\left[\begin{array}{llllll}
\lambda_{1} & \cdots & \lambda_{r} & 0 & \cdots & 0
\end{array}\right]
$$

The least significant singular values, $\lambda_{r+1}, \cdots, \lambda_{M P}$, are discarded in the low-rank approximation.

The structure of the rank-reduced estimator is shown in Fig. 7. Denoting the error of the high-rank estimator as $\mathbf{e}=\overline{\mathbf{s}}[0]-\widehat{\mathbf{s}}[0]$ the covariance matrix of the error $\mathbf{e}_{r}=\overline{\mathbf{s}}[0]-\widehat{\mathbf{s}}_{r}[0]$ for the low-rank approximation is

$$
\begin{aligned}
\mathbf{P}_{r} & =E\left\{\left(\overline{\mathbf{s}}[0]-\widehat{\mathbf{s}}_{r}[0]\right)\left(\overline{\mathbf{s}}[0]-\widehat{\mathbf{s}}_{r}[0]\right)^{H}\right\} \\
& =E\left\{\left(\mathbf{e}+\mathbf{K} \overline{\mathbf{r}}_{\omega}-\mathbf{K}_{r} \overline{\mathbf{r}}_{\omega}\right)\left(\mathbf{e}+\mathbf{K} \overline{\mathbf{r}}_{\omega}-\mathbf{K}_{r} \overline{\mathbf{r}}_{\omega}\right)^{H}\right\}
\end{aligned}
$$

In the case of no design mismatch (when a correct PSD model is used), the error $\mathbf{e}$ is orthogonal to the measurement vector $\overline{\mathbf{r}}_{\omega}$ and

$$
\begin{aligned}
\mathbf{P}_{r}= & E\left\{\mathbf{e e}^{H}+\left(\mathbf{K}-\mathbf{K}_{r}\right) \overline{\mathbf{r}}_{\omega} \overline{\mathbf{r}}_{\omega}^{H}\left(\mathbf{K}-\mathbf{K}_{r}\right)^{H}\right\} \\
= & \mathbf{P}+\left(\mathbf{K}-\mathbf{K}_{r}\right) \mathbf{R}_{\overline{\mathbf{r}}_{\omega} \overline{\mathbf{r}}_{\omega}}\left(\mathbf{K}-\mathbf{K}_{r}\right)^{H} \\
= & \mathbf{P}+\left(\mathbf{K} \mathbf{R}_{\mathbf{\mathbf { r }}_{\omega}}^{\frac{1}{2}} \overline{\mathbf{r}}_{\omega}-\mathbf{K}_{r} \mathbf{R}_{\overline{\mathbf{r}}_{\omega} \overline{\mathbf{r}}_{\omega}}^{\frac{1}{2}}\right) \\
& \times\left(\mathbf{K} \mathbf{R}_{\mathbf{r}_{\omega}}^{\frac{1}{2}} \overline{\mathbf{r}}_{\omega}-\mathbf{K}_{r} \mathbf{R}_{\mathbf{\mathbf { r }}_{\omega}}^{\frac{1}{2}} \overline{\mathbf{r}}_{\omega}\right)^{H} \\
= & \mathbf{P}+\left(\mathbf{U} \boldsymbol{\Sigma} \mathbf{Q}^{H}-\mathbf{U} \boldsymbol{\Sigma}_{r} \mathbf{Q}^{H}\right)\left(\mathbf{U} \boldsymbol{\Sigma} \mathbf{Q}^{H}-\mathbf{U} \boldsymbol{\Sigma}_{r} \mathbf{Q}^{H}\right)^{H} \\
= & \mathbf{P}+\mathbf{U}\left(\boldsymbol{\Sigma}-\boldsymbol{\Sigma}_{r}\right)\left(\boldsymbol{\Sigma}-\boldsymbol{\Sigma}_{r}\right)^{H} \mathbf{U}^{H} \\
= & \mathbf{P}+\sum_{k=r+1}^{M P} \lambda_{k}^{2} \mathbf{u}_{k} \mathbf{u}_{k}^{H}
\end{aligned}
$$


where $\mathbf{P}$ is the error covariance matrix for the full rank estimator. The mean squared error (MSE) for the rank-r estimator is then

$$
\operatorname{MSE}(r)=\operatorname{tr} \mathbf{P}_{r}=\operatorname{tr} \mathbf{P}+\sum_{k=r+1}^{M P} \lambda_{k}^{2}=\operatorname{MSE}(M P)+\sum_{k=r+1}^{M P} \lambda_{k}^{2}
$$

where $\operatorname{MSE}(M P)$ is the MSE for the high rank-MP LMMSE estimator (21) and where the last sum is the extra MSE introduced by the low-rank approximation.

\section{Rank of the NBI}

The theory of essentially time- and bandlimited signals tells how many dimensions are needed in the low-rank approximation. In [6] it is shown that the number of most significant eigenvalues of a time- and bandlimited signal equals $2 B T+1$ where $B$ is the one-sided bandwidth of a baseband signal and $T$ is the length of the observation interval. Here, $B$ is given by the PSD, $P_{S_{b}}(f)$, of the NB signal $s_{b}(t)$.

We relate the bandwidth of the NB signal to the OFDM subcarrier spacing as $2 B=b f_{s} / N$, where $b$ is a number, as sketched in Fig. 3, representing the double-sided bandwidth of $s_{b}(t)$. Our observation interval, which we consider for NBI cancellation, is one OFDM frame stripped from its cyclic extension, giving $T=N / f_{s}$. Hence, the effective rank of the NB signal is

$$
r_{\text {eff }}=2 B T+1=b+1
$$

which indicates that it is possible to reduce the rank of the estimator from $M P$ down to $b+1$ and maintain most of its performance.

However, for scenarios in which NBI cancellation is important the background noise power is typically much lower than the NBI power. For this reason a slightly higher rank than $r_{\text {eff }}$ need often to be used in order not to sacrifice cancellation performance. For the NBI cancellation examples shown in this paper, we have used

$$
r_{\text {low }}=r_{\text {eff }}+2=b+3
$$

as a quite conservative rule of thumb. This results in virtually the same performance for the rank reduced canceller as for the full-rank canceller except for scenarios with very high SNR. Then, the rank may have to be slightly higher in order not to sacrifice any performance.

Fig. 8 shows the power in the singular values $\lambda_{k}$, relative to the most significant $\lambda_{1}$, for some different bandwidths of the NB signal represented by its time-bandwidth product $b$. This is in good agreement with (46). The PSD shape was flat and $b$ subcarriers wide. In this example we used $M=11$ subcarriers as measurement tones closest to the subcarrier $k_{0}$ with most interference, $\omega=\left\{k_{0}-5, \ldots, k_{0}+5\right\}$, using either three consecutive frames (solid curves) or only one frame (dashed curves). This means $P=3$ frames $\left(L_{n}=L_{p}=1\right)$ or $P=1$ frame $\left(L_{n}=L_{p}=0\right)$, giving a rank of either $M P=33$ or $M P=11$, respectively, for these two different full-rank estimators (21). In both cases the NBI to background noise power ratio was $20 \mathrm{~dB}$.

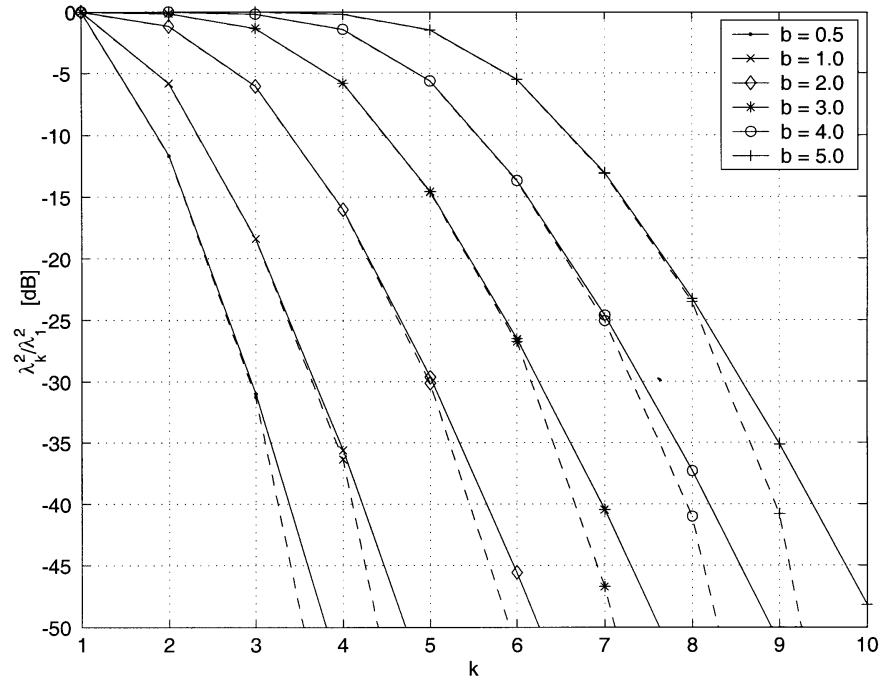

Fig. 8. Relative powers for the singular values, $\lambda_{k}^{2} / \lambda_{1}^{2}$, for different time-bandwidth products, $b=2 B T$. Solid lines represent 33 measurement tones taken from three OFDM frames, dashed lines 11 tones in one frame.

Table I shows the corresponding estimator energies in the first $b+1, b+2$ and $b+3$ dimensions relative to the total energy in all 33 dimensions (with $P=3$ ) for different NB signal bandwidths $b$. The corresponding relative energies when using one frame with eleven measurement tones, $(P=1, M=11)$ are strictly, but only slightly, closer to one; see Fig. 8 in which the dashed curves drops increasingly quicker than the solid for the singular values that exceed $r_{\mathrm{eff}}=b+1$.

For the improper NB signal model (3) the total bandwidth spanned doubles (when $f_{c}>b / 2$ ) and the corresponding time bandwidth product becomes $2 B T=2 b$. However, baseband systems like DSL can utilize only half of the available spectrum, $0<f<f_{s} / 2$, for (unique) data transmission since the other half obeys complex symmetry. Therefore, the effective rank of the NB signal in the half frequency band of interest remains $r_{\text {eff }}=b+1$. This means that the rule of thumb (47), i.e., same rank, can be used also for the improper NB signal cases (baseband scenarios) provided that nonrectangular receiver windowing is performed. However, the usually high SNR for many DSL applications may require a slightly higher rank $(r=b+4$ or $r=b+5$ ), as mentioned earlier.

The receiver windowing suppresses the NBI leakage between the complex symmetric positive and negative frequency halves. Without receiver windowing spectral leakage from one frequency half to the other requires that the rank must be increased by one, for cases where the rank is selected as $r \geq b+3$, to obtain a similar good performance (for a given $r$ ) as when receiver windowing is used.2

\section{Cancellation Complexity}

By considering that the SVD computation can be performed offline, typically during the installation of the receiver, the estimator coefficients for a specific application scenario can be downloaded, or stored, into the receiver prior its operation. However, it is also possible to recompute new coefficients and

\footnotetext{
${ }^{2}$ The poorer performance achieved when $r<b+3$ is essentially the same with and without nonrectangular receiver windowing.
} 
TABLE I

RElative Estimator ENERgIES With $M P=33$ MEASUREMENT TONES

\begin{tabular}{c||c|c|c|c|c|c}
\hline$\sum_{k=1}^{r} \lambda_{k}^{2} / \sum_{k=1}^{33} \lambda_{1}^{2}$ & $b=0.5$ & $b=1.0$ & $b=2.0$ & $b=3.0$ & $b=4.0$ & $b=5.0$ \\
\hline \hline$r=\lceil b+1\rceil$ & 0.99925 & 0.98841 & 0.98714 & 0.98760 & 0.98834 & 0.98908 \\
\hline$r=\lceil b+2\rceil$ & 1.00000 & 0.99978 & 0.99945 & 0.99923 & 0.99908 & 0.99899 \\
\hline$r=\lceil b+3\rceil$ & 1.00000 & 1.00000 & 0.99997 & 0.99997 & 0.99995 & 0.99994 \\
\hline
\end{tabular}

TABLE II

COMPLEXITy REDUCTIONS USING MP MEASUREMENT TONES

\begin{tabular}{c||c|c|c|c|c|c}
\hline $\begin{array}{c}\text { Reduction of } \\
\text { multiplications }(\%) \\
M P=33, M P=11\end{array}$ & $b=0.5$ & $b=1.0$ & $b=2.0$ & $b=3.0$ & $b=4.0$ & $b=5.0$ \\
\hline \hline$r=\lceil b+1\rceil$ & 93,81 & 93,81 & 90,72 & 86,62 & 83,53 & 79,43 \\
\hline$r=\lceil b+2\rceil$ & 90,72 & 90,72 & 86,62 & 83,53 & 79,43 & 76,34 \\
\hline$r=\lceil b+3\rceil$ & 86,62 & 86,62 & 83,53 & 79,43 & 76,33 & 73,24 \\
\hline
\end{tabular}

download to the receiver if the application specifications should change. With this perspective, the continuous computational complexity, during receiver operation, can be lowered with the low-rank approximation as described below.

We formulate the rank- $r$ NBI canceller (35) as [18]

$$
\widehat{\mathbf{s}}_{r}[0]=\left(\sum_{k=1}^{r} \lambda_{k} \mathbf{u}_{k} \mathbf{v}_{k}^{H}\right) \overline{\mathbf{r}}_{\omega}=\sum_{k=1}^{r} \mathbf{p}_{k}\left\langle\mathbf{v}_{k}, \overline{\mathbf{r}}_{\omega}\right\rangle
$$

where $\mathbf{p}_{k}=\lambda_{k} \mathbf{u}_{k}$ and $\mathbf{v}_{k}$ are vectors of length $N$ and $M P$, respectively. Each inner product, $\left\langle\mathbf{v}_{k}, \overline{\mathbf{r}}_{\omega}\right\rangle$, requires $M P$ multiplications, i.e., $r M P$ multiplications in total. The linear combination of $r$ vectors each of length $N$ requires $r N$ multiplications. In each frame, $N$ subcarriers are estimated simultaneously giving

$$
\frac{r M P+r N}{N}=r\left(1+\frac{M P}{N}\right)
$$

multiplications per subcarrier.

The rank reduction for lowering the complexity is most valuable when more measurement tones are used than the time-bandwidth product specifies. The original estimator (21) requires $M P$ multiplications per subcarrier resulting in a complexity reduction of $1-r(1 / M P+1 / N)$ using the low-rank approximation. Table II shows examples of the complexity reduction using the parameters from the previous section and $N=256$ subcarriers.

\section{Performance Analysis}

We evaluate the performance for an OFDM system with $N=$ 256 subcarriers ( $N=2048$ (FFT size 4096) in the DMT-VDSL scenario in Section IV-G) and we use an NB signal whose center frequency, $f_{c}$, is located just in between two subcarriers, \#77 and $\# 78$, i.e., $f_{c} / f_{s} N=77.5$, which causes a large spectral leakage onto all subcarriers even if the actual NB signal would not have any bandwidth $\left(b_{\text {true }}=0.0\right)$. In all sections except Section IV-F we use only the current OFDM frame in the estimation of the
NBI ( $\left.P=1, L_{n}=L_{p}=0\right)$. Thus, the same performance and the same estimator coefficients will be obtained if the interference would be located in between any other two subcarriers than the one selected here, as discussed in Section III-A.

The average power of the interference before suppression is 0 $\mathrm{dBm} / \mathrm{Hz}$ in all cases. As before, the 11 subcarriers closest to the peak of the interference are used as measurement tones, $\omega=$ $\{74, \ldots, 84\}$. We use a sampling frequency of $f_{s}=10 \mathrm{MHz}$ which gives a subcarrier spacing of $39 \mathrm{kHz}$.

The average error, $\mathbf{e}=\overline{\mathbf{s}}[0]-\widehat{\mathbf{s}}[0]$, has covariance matrix

$$
\begin{aligned}
\mathbf{P}=E\left\{\mathbf{e e}^{H}\right\}=\mathbf{K} \mathbf{R}_{\overline{\mathbf{r}}_{\omega} \overline{\mathbf{r}}_{\omega}} \mathbf{K}^{H}+\mathbf{R}_{\overline{\mathbf{s}}[0] \overline{\mathbf{s}}[0]} \\
-\mathbf{K R}_{\overline{\mathbf{r}}_{\omega} \overline{\mathrm{s}}[0]}-\mathbf{R}_{\overline{\mathbf{s}}[0] \overline{\mathbf{r}}_{\omega}} \mathbf{K}^{H}
\end{aligned}
$$

when the model parameters deviate from the true. When the modeled and true parameters agree, (50) reduces to

$$
\mathbf{P}=\mathbf{R}_{\overline{\mathbf{s}}[0] \overline{\mathbf{s}}[0]}-\mathbf{K R}_{\overline{\mathbf{r}}_{\omega} \overline{\mathrm{s}}[0]} .
$$

In the case of rank reduction, $\mathbf{P}_{r}$ and $\mathbf{K}_{r}$ replaces $\mathbf{P}$ and $\mathbf{K}$, respectively. The MSE, the PSD of the residual error, is then $\operatorname{diag}(\mathbf{P})$ using either (50) or (51). The performance evaluations which will be presented in Sections IV-A-F are based on the analytical expressions (50) and (51), which agree with simulation results using Monte Carlo methods (not included in these sections but which are used for the DMT-VDSL application scenario in Section IV-G and in Section IV-H).

First, we evaluate the performance in an ideal case where we show the level of performance that can be achieved if the NB signal's PSD is modeled perfectly by using (51). Secondly, we evaluate the performance in a mismatched case by using (50), where we assume that we have quite poorly modeled the NB signal and also reduced the rank too much. Thereafter, we examine the robustness against frequency errors and bandwidths of the NB signal and show the effects of nonrectangular windowing and by using several OFDM frames for the canceller. Where indicated, a raised-cosine window is used with $\mu=6$ extra samples within the $\mathrm{CE}$ for windowing. 


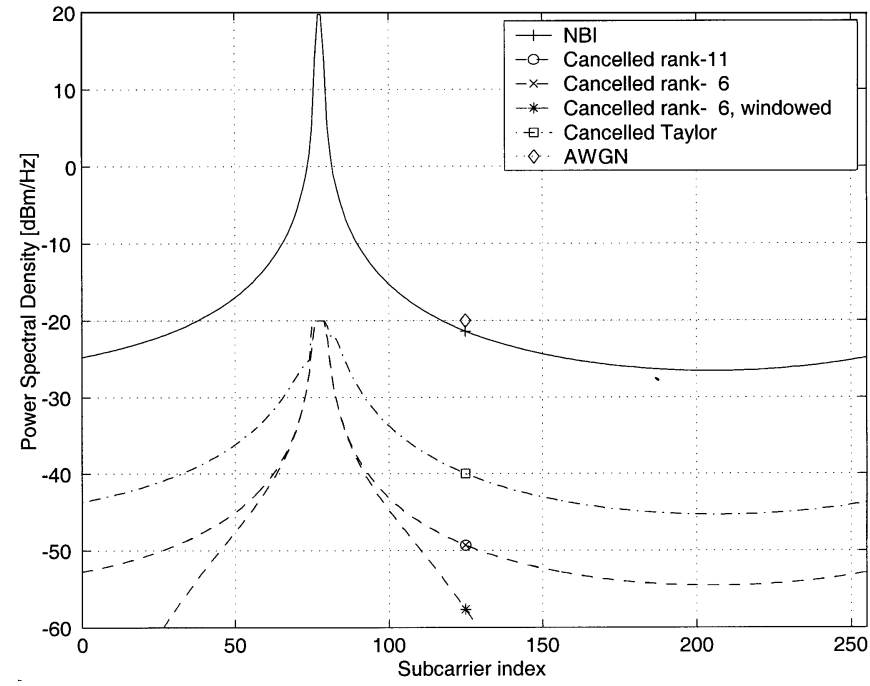

Fig. 9. An ideal case; residual error PSDs after NBI cancelling. NB signal bandwidth $b=2.5$ subcarriers. The rank-11 residual (circle marker) and the rank-6 residual (cross marker) are indistinguishable in this scale. AWGN at -20 $\mathrm{dBm} / \mathrm{Hz}$.

\section{A. Performance for an Ideal Case}

In the ideal case all model parameters agree with the true NB signal. Here, we use a rectangular PSD shape for the NB signal bandwidth that overlaps $b=2.5$ OFDM subcarriers.

Fig. 9 shows the residual error PSD, $\operatorname{diag}(\mathbf{P})$, before and after NBI suppression using an LMMSE rank-11 (circle marker, o) and a rank-6 estimator (cross marker, $\times$ ). These curves are inseparable in this scale and lie below the background noise at $-20 \mathrm{dBm} / \mathrm{Hz}$ on all subcarriers. The windowed case (star marker, $*$ ) uses $\mu=6$ samples for windowing. As a comparison, Fig. 9 shows also the suppression performance by a Taylor-parametrized RFI canceller using seven parameters (square marker, $\square$ ) [13].

\section{B. Performance for a Model Mismatched Case}

In this evaluation we have a design mismatch between the true and modeled PSD of the NB signal. A rectangular PSD shape is used in our model with a DSB bandwidth that overlaps $b=$ 3.0 OFDM subcarriers, but the true NB signal has a Gaussianshaped PSD which overlaps $b_{\text {true }}=2.0$ subcarriers (taken as the $-6 \mathrm{~dB}$ DSB-bandwidth from the PSD peak). Further, we have an error in the modeled center frequency of the NB signal, $N\left|\widehat{f}_{c}-f_{c}\right| / f_{s}=0.2$ subcarriers.

Fig. 10 shows the residual error PSD, $\operatorname{diag}(\mathrm{P})$ using (50), before and after the cancellation. For this case the suppression of the NBI on subcarriers away from $f_{c}$ is still quite effective but the residual errors on a few tones close to $f_{c}$ are slightly above the background noise floor at $-20 \mathrm{dBm} / \mathrm{Hz}$.

In this case, the rank-11 estimator (circle marker, o, in Fig. 10) performs slightly better than the rank-6 estimator (cross marker, $x$ ). In this case the effective rank is 4 and the rule of thumb in (47) suggests $r_{\text {low }}=6$. The Taylor-based RFI canceller [13] (square marker, $\square$ ) is shown for comparison.

The increased residual errors compared to the ideal case depends mainly on that the Gaussian tails on the true PSD are not

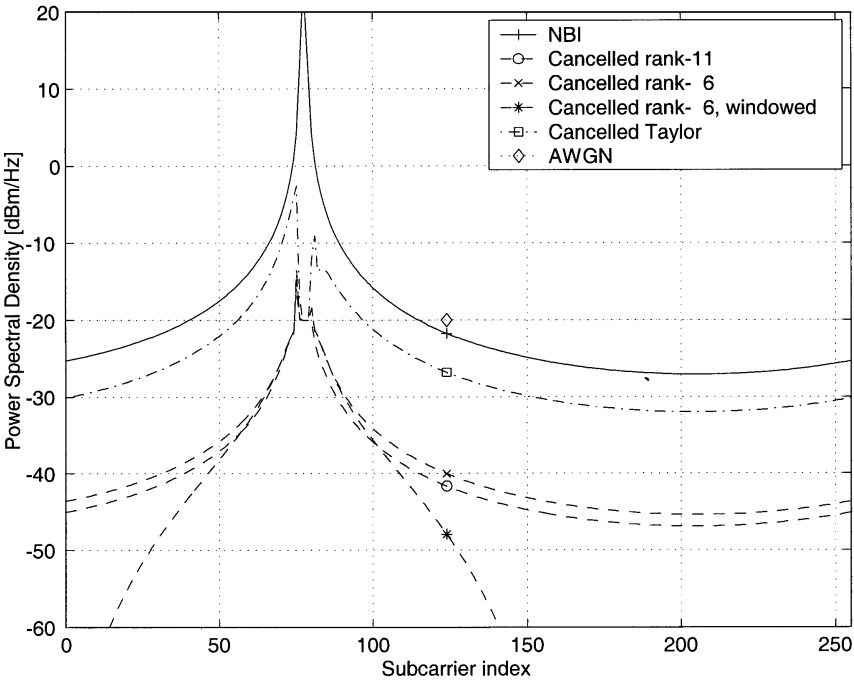

Fig. 10. A mismatched case; residual error PSDs after NBI cancelling. NB signal PSD Gaussian shaped, bandwidth $b_{\text {true }}=2.0$ (at $-6 \mathrm{~dB}$ PSD). Modeled with $b=3.0$ flat shaped. Center-frequency error: $\Delta f_{c}=0.2$ subcarriers. AWGN at $-20 \mathrm{dBm} / \mathrm{Hz}$

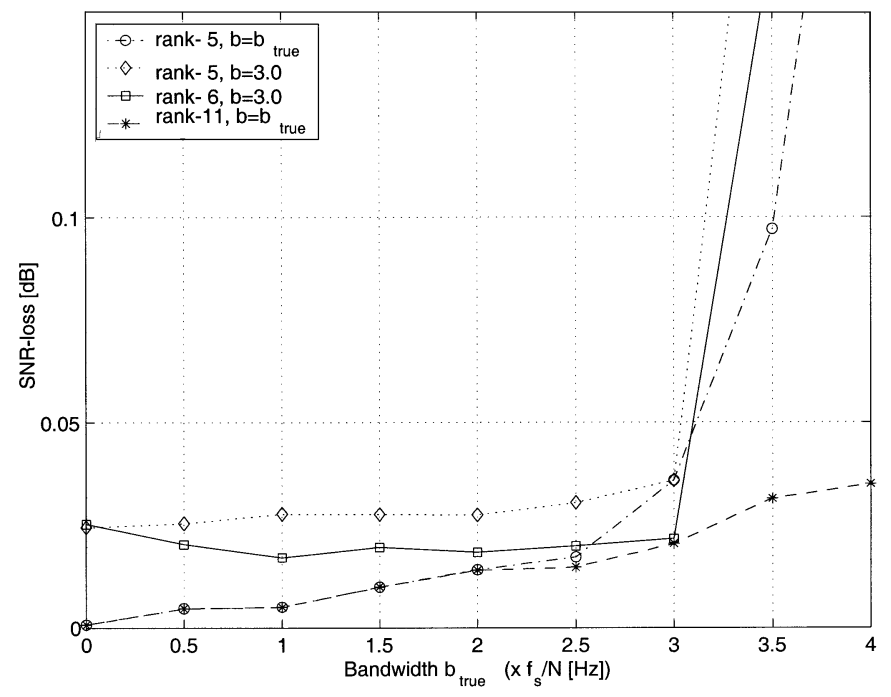

Fig. 11. Average SNR loss as a function NB signal bandwidth, $b_{\text {true }}$. The NB signal has flat PSD shape. The rank-5 (o) and rank-11 $(*)$ estimators have a correctly modeled bandwidth, $b=b_{\text {true }}$. The rank-5 $(\diamond)$ and rank-7 ( $\square$ ) estimators have a fixed modeled bandwidth, $b=3.0$.

covered by the rectangular-modeled PSD. The NBI from these uncovered PSD tails are not cancelled.

\section{Varying the Bandwidth of the NB Signal}

Using (50), we define the average SNR loss as

$$
\begin{aligned}
\text { SNR-loss } & \triangleq \frac{1}{N-M} \sum_{\substack{k=0 \\
k \notin \omega}} \frac{E\left\{\left|\bar{v}_{k}\right|^{2}+\left|\bar{s}_{k}-\widehat{s}_{k}\right|^{2}\right\}}{E\left\{\left.\bar{v}_{k}\right|^{2}\right\}} \\
& =1+\frac{\operatorname{tr}\left(\mathbf{M}_{\omega} \mathbf{P}_{r}\right)}{(N-M) \sigma^{2}}
\end{aligned}
$$

where $\mathbf{M}_{\omega}$ is a diagonal matrix with zeros at the indexes corresponding to the measurement tones, $\omega$, and ones elsewhere. Fig. 11 shows the average SNR loss, with exclusion of the silent 
measurement tones $\omega$, for different bandwidths of the NB signal. The frequency axis is scaled into the OFDM subcarrier spacing, $f_{s} / N$, where $b_{\text {true }}$ is the true NB signal bandwidth. If the measurement tones $\omega$ are also included in the SNR-loss calculation, then $\mathbf{M}_{\omega}=\mathbf{I}$, which gives

$$
\text { SNR loss }=1+\frac{\operatorname{tr}\left(\mathbf{P}_{r}\right)}{N \sigma^{2}}
$$

The average SNR loss before NBI cancellation is $20 \mathrm{~dB}$. Further, the center-frequency error, $N\left|\hat{f}_{c}-f_{c}\right| / f_{s}$, is zero and we used a rectangular shape both for the modeled and the true NB signal's PSD.

For the rank-5 and the rank-11 estimators (with circle-, o, and star-, *, markers in Fig. 11, respectively) we modeled the bandwidth correctly, $b=b_{\text {true }}$. This rank- 5 estimator is capable of giving the same performance as the rank-11 estimator for $b \leq 2.0$. That agrees with the rule of thumb in (47). For $b>2.0$, the rank-5 is too low and it suffers increasingly higher SNR degradation.

With the rank-6 estimator we have fixed the modeled bandwidth to 3.0 subcarriers wide. This is capable of giving good performance for NB signals that have smaller bandwidth. Since it performs almost as well as if we knew its true bandwidth it tells how to make a robust estimator if we do not know the bandwidth of the interfering signal. The modeled PSD should be as wide as the maximal expected width (using a flat PSD-shape). Then, in order to reduce the cancellation complexity the rank can be reduced to $b+3$. This is the case with the rank- 6 estimator which is designed to model an NB signal with a bandwidth up to $b=3.0$ subcarriers. It gives good and consistent performance for $0 \leq b_{\text {true }} \leq b$. Then, when $b_{\text {true }}>b$ the modeled bandwidth is too small resulting in an increasingly higher SNR loss.

There are two prices to pay for selecting a too large bandwidth in the model. One is that it performs slightly worse for smaller bandwidths as an estimator with a more correctly (narrower) modeled bandwidth. The other is that it is not possible to reduce the rank as much for a large modeled bandwidth as for a low modeled bandwidth, without sacrificing performance.

An example of performance loss due to a bit more aggressive rank-reduction is the rank-5 estimator with fixed modeled bandwidth, $b=3.0$, (with diamond, $\diamond$, markers) in Fig. 11. Here, we reduced the rank below the rule of thumb $r_{\text {low }}=6$. This is why it performs slightly worse than the equivalent rank- 6 estimator for $b<3.0$ by suffering slightly higher SNR degradation.

\section{Impact of Center-Frequency Errors}

Fig. 12 shows the impact of center-frequency errors, $\Delta f_{c} \triangleq$ $N\left|\hat{f}_{c}-f_{c}\right| / f_{s}$, using (50) and (52). In this performance evaluation both the modeled and the true PSD shapes are flat. The true signal has a baseband (DSB) bandwidth of $b_{\text {true }}=2.0$.

The LMMSE rank-5 and rank-11 estimators (with circle, o, and star, $*$, markers in Fig. 12, respectively) are both designed with the same modeled PSD bandwidth as the true, $b=b_{\text {true }}=$ 2.0. This gives no room for a frequency-shift of the NB signal's PSD from its modeled (which corresponds to a center-frequency error). Therefore, they perform worse than the rank-5 and rank-6 estimators that have $b=2.5>b_{\text {true }}$ (square, $\square$, and triangle, $\nabla$,

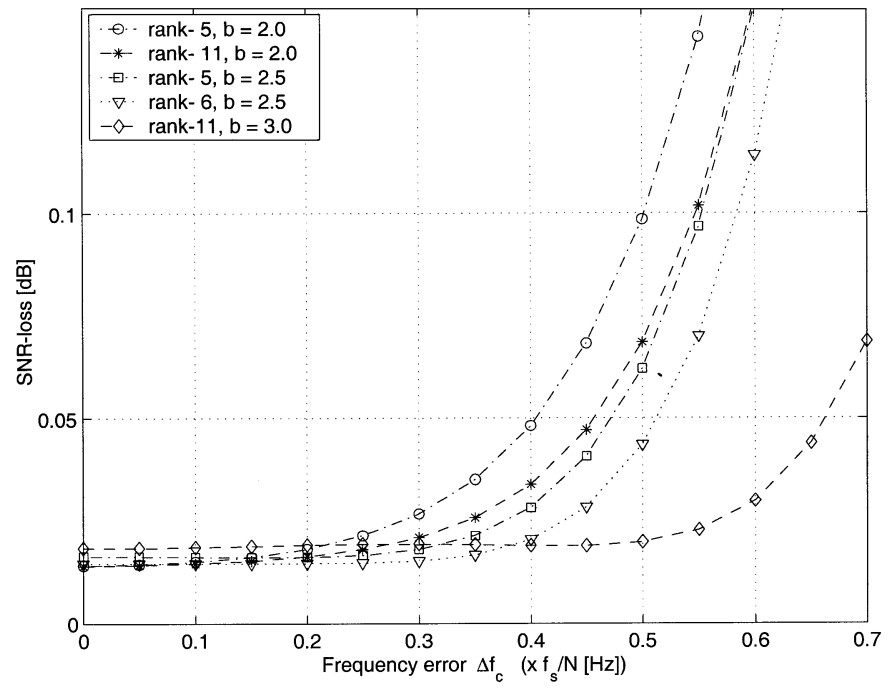

Fig. 12. Average SNR-loss as a function of center-frequency error $\Delta f_{c}$. The NB signal has a flat PSD-shape. True NB signal bandwidth is $b_{\text {true }}=2.0$ and modeled with $b$.

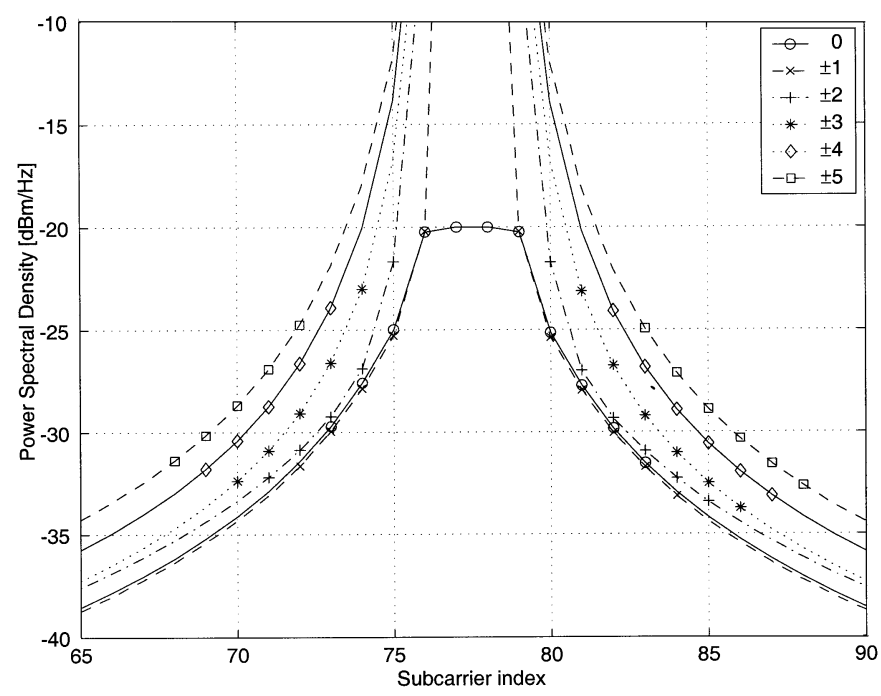

Fig. 13. Residual error PSD after NBI cancelling for different placements of the measurement tones. The markers indicate the location of the 11 measurement tones in each case.

markers in Fig. 12, respectively). The latter estimators can better allow a frequency shift than their equivalences with $b=b_{\text {true }}$, but the rank-5 estimator suffers from a slightly higher SNR degradation than the rank-6 estimator due to its lower rank.

Finally, the rank-11 estimator with $b=3.0$ (diamond, $\diamond$, marker in Fig. 12) gives low and consistent SNR loss for all center-frequency errors in the range $0 \leq \Delta f_{c} \leq 0.5$. This is because frequency errors less than 0.5 subcarriers on the 2.0 subcarriers-wide PSD is still within the modeled PSD bandwidth of 3.0 subcarriers-wide NB signal.

\section{E. Placement of Measurement Tones}

The placement of the measurement tones affects the suppression performance. Fig. 13 shows the residual error PSD after NBI-cancelling using (51) and 11 measurement tones (in $P=1$ frame) with different distances from the center peak of the NB signal. The markers indicate their frequency locations for each 


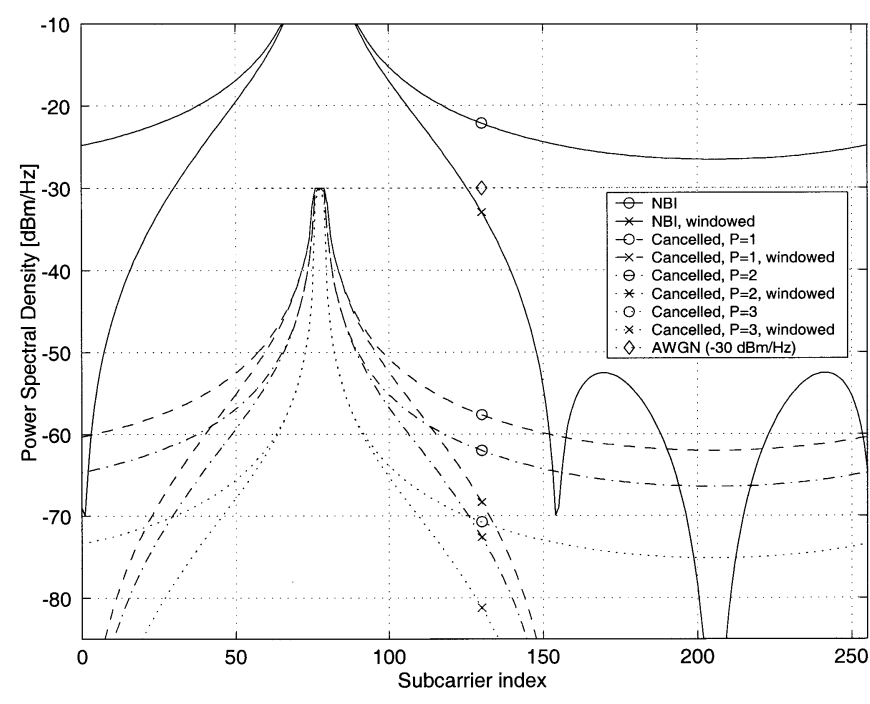

Fig. 14. Residual error PSDs with/without windowing $(\mu=6 / \mu=0)$ and using several frames in NBI estimation. $P=1$ uses current frame only $\left(L_{n}=\right.$ $\left.L_{p}=0\right) . P=2$ uses current and one previous frame $\left(L_{n}=1, L_{p}=0\right)$. $P=3$ uses previous, one current and one frame ahead $\left(L_{n}=L_{p}=1\right)$. AWGN at $-30 \mathrm{~dB}$.

case. The NB signal bandwidth is $b=2.0$ with center frequency located at $f_{c} N / f_{s}=77.5$. The average NBI power is $0 \mathrm{dBm} / \mathrm{Hz}$ and background noise power is $-20 \mathrm{dBm} / \mathrm{Hz}$.

When the measurement tones are placed around and covering the interference peak (indicated with circle, o, markers in Fig. 13) the residual interference is suppressed below the background noise floor at $-20 \mathrm{dBm} / \mathrm{Hz}$ on all subcarriers. If the measurement tones are placed around, but not covering the peak (corresponding to cases $\pm 1, \ldots, \pm 5$ ), a strong residual peak will remain after cancellation along the gap between the measurement tones. In this latter case, tones away from the peak show only marginally better performance $( \pm 1)$ or worse performance $( \pm 2, \ldots, \pm 5)$ than the case where the measurement tones are covering the peak entirely (0).

In order to be cooperative it is unwise to use the peak tones for data transmission regardless whether they are used as measurement tones or not. Hence, it is often best to use the peak interference tones as measurements. Then, good performance is achieved at the same time as the measurement tones are less likely conflicting with subcarriers that can be used for data transmission. Measurement tones at the peak will also pick up more of the inherent dimensionality in the interference and less background noise than those placed further away from the peak.

\section{F. Effects of Windowing and Using Several Frames}

Fig. 14 shows the effects [by using (51)] of windowing with a raised-cosine shape in combination with the cancellation and the effects of using more than one $(P>1)$ OFDM frame for the estimation of the $\mathrm{NBI}(N=256)$. In this example the white noise level is $-30 \mathrm{dBm} / \mathrm{Hz}$. Using two frames $P=2$ for NBI measurement (current plus one previous frame, $L_{n}=1, L_{p}=0$ ) increases the NBI suppression with more than $4 \mathrm{~dB}$ on all subcarriers (compared to using one frame), except for the very few subcarriers under the NBI-peak. Using $P=3$ frames (current frame, one previous, and one frame ahead $\left(L_{n}=L_{p}=1\right)$
TABLE III

HAM BANDS IN EUROPE (kHz)

\begin{tabular}{l||l|l|l|l|l|l}
\hline Band start & 1810 & 3500 & 7000 & 10100 & 14000 & 18068 \\
\hline Band stop & 2000 & 3800 & 7100 & 10150 & 14350 & 18168 \\
\hline
\end{tabular}

TABLE IV

SELECTED DMT-VDSL PARAMETERS

\begin{tabular}{l||r}
\hline Number of subcarriers, $N$ & 2048 \\
\hline Sampling frequency, $f_{s}$ & $17.664 \mathrm{MHz}$ \\
\hline Subcarrier spacing, $f_{s} /(2 N)$ & $4.3125(\triangleq \Delta f) \mathrm{kHz}$ \\
\hline True RFI-bandwidth, $b_{\text {true }} f_{s} /(2 N)$ & $5.0(=1.16 \Delta f) \mathrm{kHz}$ \\
\hline Modeled RFI-bandwidth, $b f_{s} /(2 N)$ & $8.625(=2 \Delta f) \mathrm{kHz}$ \\
\hline Center-frequency, $f_{c}$ & $3525 \mathrm{kHz}$ \\
\hline Center-frequency error, $\widehat{f}_{c}-f_{c}$ & $2.156(=0.5 \Delta f) \mathrm{kHz}$ \\
\hline Window size, $\mu$ & 70 samples \\
\hline
\end{tabular}

increases the suppression with more than $10 \mathrm{~dB}$ on most subcarriers, except for the very few tones under the peak.

In this example the cyclic extension was $C E=10$ samples long (including the windowing samples). A longer $\mathrm{CE}$ reduces slightly the effect of using several frames while a shorter CE increases the effect slightly. This is because the correlation between different OFDM frames is affected by the length of the $\mathrm{CE}$ (distance between frames).

Nonrectangular windowing alone reduces the NBI on subcarriers far away from $f_{c}$ resulting in fewer subcarriers needing NBI cancellation. Where indicated in Fig. 14, $\mu=6$ extra samples are used from the CE for windowing. Using more samples reduces the number of subcarriers that need NBI cancellation even more, but at the expense of a reduced efficiency. The interference is cancelled by a similar amount with/without nonrectangular windowing, but because of the windowing, the interference is suppressed to a lower level on subcarriers a bit away from the peak.

\section{G. Application Example: RFI Cancellation in DMT-Based VDSL}

The proposed canceller can be incorporated naturally in the receiver structure for a DMT-based VDSL modem specified by ETSI [4] to cancel RFI from amateur radio. The functional requirements in the DSL standards specifies a mandatory avoidance of transmission in the amateur radio HAM bands, see Table III, in order to avoid egress in these bands. ${ }^{3}$ Hence, silent measurement tones for the RFI canceller are available in these bands without sacrificing usable bandwidth.

To simulate RFI noise cancellation in VDSL, we used a DMT-VDSL system specified by ETSI. See Table IV for some of the system and RFI parameters. The environment was a 1000 m-long TP1 cable [19] and the noise consisted of 25 self-FEXT disturbers plus AWGN at $-140 \mathrm{dBm} / \mathrm{Hz}$. We placed the RFI-signal's center frequency close the edge of the second HAM band at $3525 \mathrm{kHz}$ and adjusted it in between two subcarriers. The average RFI power was equal to the average received VDSL signal power (SIR $0 \mathrm{~dB}$ ). A center-frequency error of $\widehat{f_{c}}-f_{c}=2.156 \mathrm{kHz}$ was used, corresponding to half of the subcarrier spacing $(4.3125 \mathrm{kHz})$ and $b=2, b_{\text {true }}=1.16$, see

\footnotetext{
${ }^{3}$ Due to sidelobe leakage at the Tx, a few extra subcarriers close to the HAM band edges may also have to be turned off.
} 


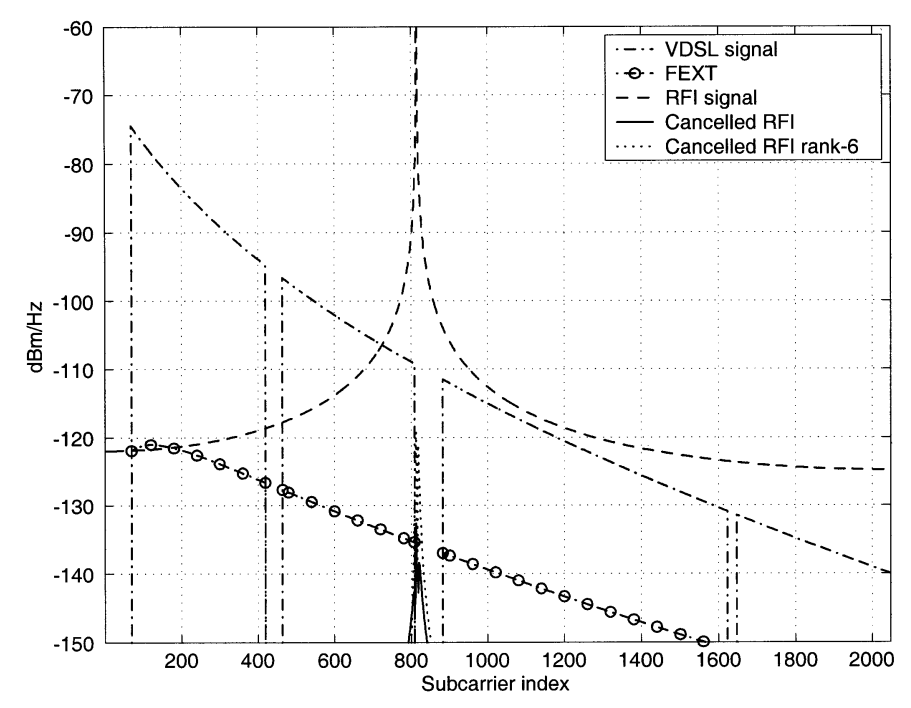

Fig. 15. RFI cancellation in a DMT-VDSL system with 2048 subcarriers.

Table IV. For the canceller design, eleven measurement tones $(M=11)$ were used in one DMT frame $(P=1)$ and a raised cosine receiver window of size $\mu=70$ samples was used. With $P=1$, windowing and the robust-modeled RFI bandwidth, the same estimator coefficients used in this example can also be used to cancel RFI successfully at any other frequency location within the HAM bands. With rank reduction the number of (complex) multiplications that are required continuously during operation was reduced from 11 to 6 per subcarrier. Without the receiver windowing a rank of 7 (instead of 6) would be needed to obtain the same performance. Note that the DFT length is doubled, $2 N$, due to the baseband signalling but that only half of the subcarriers, $0 \ldots N-1$, are of interest.

Fig. 15 shows the Monte Carlo simulation results using the proposed canceller. The resulting interference is suppressed below the background AWGN floor at $-140 \mathrm{dBm} / \mathrm{Hz}$, except for only a few subcarriers inside the HAM band when the reduced rank- 6 canceller is used. Because of the very high average SNR in this application (background AWGN floor at $-140 \mathrm{dBm} / \mathrm{Hz}$ ), the amount of suppression is higher than in previous examples but the performance loss due to rank reduction becomes slightly more visible. However, increasing the rank from 6 to 7 will suppress the remaining residual power to below the background noise floor.

Fig. 16 shows the resulting SNR with/without RFI and cancellation. The two SNR curves with RFI and cancelling $(x$ and + markers) are indistinguishable from the SNR curve without any RFI present (o marker). The SNR curve with RFI but no cancellation ( $\diamond$ marker) shows significant SNR-loss on most subcarriers. Observe in this application example that all subcarriers within the HAM bands are silent and excluded when calculating the SNR loss. Table $\mathrm{V}$ shows the corresponding average symbol error rate (SER) to the curves in Fig. 16. A simple, but conservative, bitloading algorithm was used

$$
\operatorname{bits}_{k}=\left\lfloor\log _{2}\left(1+\frac{\mathrm{SNR}_{k}}{\Gamma}\right)\right\rfloor
$$

where bits $_{k}$ is the number of bits assigned to subcarrier $k$ and where $\Gamma=9.8 \mathrm{~dB}$ is the SNR gap [20] which results in an

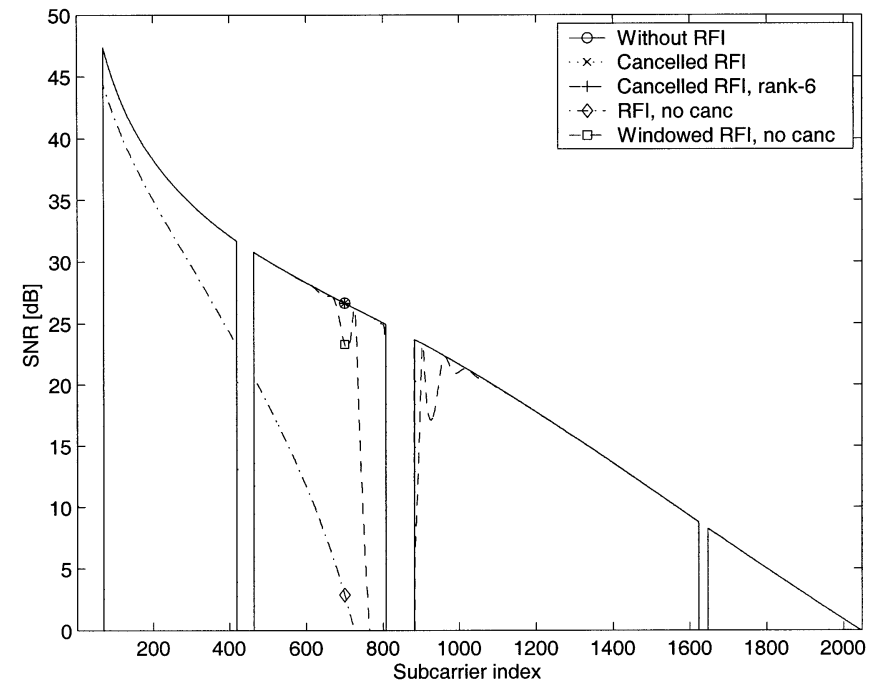

Fig. 16. Average SNR with/without RFI and cancellation.

SER of less than $10^{-7}$ on all subcarriers without RFI. In this example the resulting average SER was obtained by using the analytical SER expression for ( $\left.2^{\text {bits }_{k}}\right)$-size QAM constellations on AWGN channels [21], and by assuming that the residual RFI noise is Gaussian distributed on each subcarrier. A comparison with Monte Carlo simulated and analytical SER is shown in Table VI where the target SER was increased to less than $10^{-3}$ and where the number of simulated bit loaded and RFI cancelled DMT frames was $10^{5}$.

To reduce the number of multiplications needed for each tone more substantially it is possible allow a residual peak within the silent HAM band along with a gap between the measurement tones, as shown in Section IV-E. Then, in order to obtain an increased complexity reduction the modeled bandwidth, the number of measurement tones and the rank can all be reduced at the expense of a decreased robustness.

\section{H. NBI Cancellation Without Using Silent Tones}

In this section we show a performance example of cancelling inband NBI in an OFDM system but without using any silent measurement tones. In this case the OFDM signal is modeled as part of the background noise, as discussed in Section III. Table VII shows the selected OFDM and NBI parameters and Fig. 17 shows typical PSD levels that can be expected. In this example the average NBI power was equal to the OFDM signal power $(0 \mathrm{dBm} / \mathrm{Hz}$, SIR $0 \mathrm{~dB})$ and the SNR was $30 \mathrm{~dB}$ (AWGN at $-30 \mathrm{dBm} / \mathrm{Hz}$ ). The average SNR loss was reduced from $30.0 \mathrm{~dB}$ without cancellation to $10.7 \mathrm{~dB}$ by using the canceller. With rank reduction, the number of multiplications per tone was reduced from 11 (rank 11) to 2.1 (rank 2) with a minor performance loss (the SNR loss increased from 10.7 to $10.8 \mathrm{~dB}$ ). Note that the efficient rank is 2 in this case $(b=1$ in (46)). Observe also that all tones are included in the SNR-loss calculation $\left(\mathbf{M}_{\omega}=\mathbf{I}\right.$ in (52)). As in previous examples, the NBI is suppressed below the background measurement noise, which in this case is dominated by the OFDM signal itself.

A standard block coding scheme can effectively correct symbol errors that are caused by the remaining NBI after cancellation (which are most frequent at the tones close to 
TABLE V

Average SER With/Without RFI and CANCELlation. SimUlated RFI CANCELLATION, ANALYTICAL SER EVALUation. TARgEt SER $\leq 10^{-7}$

\begin{tabular}{c|c|cc|cc}
\cline { 2 - 6 } & \multirow{2}{*}{ Without RFI } & \multicolumn{2}{c|}{ Cancelled RFI } & \multicolumn{2}{c}{ No RFI cancellation } \\
& & Full rank & Rank-6 & No windowing & With windowing \\
\hline SER & $5.93 \times 10^{-9}$ & $7.25 \times 10^{-9}$ & $4.18 \times 10^{-8}$ & 0.33 & 0.038 \\
\hline
\end{tabular}

TABLE VI

Average SER With/Without RFI ANd CANCEllation. ANALYTical VERsus Simulated CANCELlation ANd SER Evaluation. TARGEt SER $\leq 10^{-3}$

\begin{tabular}{c||c|cc|cc}
\hline \multirow{2}{*}{ SER } & \multirow{2}{*}{ Without RFI } & \multicolumn{2}{|c|}{ Cancelled RFI } & \multicolumn{2}{c}{ No RFI cancellation } \\
& & Full rank & Rank-6 & No windowing & With windowing \\
\hline Analytical & $6.51 \times 10^{-5}$ & $6.57 \times 10^{-5}$ & $6.76 \times 10^{-5}$ & 0.364 & 0.0405 \\
\hline Simulated & $6.47 \times 10^{-5}$ & $6.53 \times 10^{-5}$ & $6.70 \times 10^{-5}$ & 0.398 & 0.0442 \\
\hline
\end{tabular}

TABLE VII

SELECTED OFDM PARAMETERS

\begin{tabular}{l||r}
\hline Number of subcarriers, $N$ & 256 \\
\hline Sampling frequency, $f_{s}$ & $10 \mathrm{MHz}$ \\
\hline Subcarrier spacing, $f_{s} / N$ & $39(\triangleq \Delta f) \mathrm{kHz}$ \\
\hline True NBI-bandwidth, $b_{\text {true }} f_{s} / N$ & $9.8(=0.25 \Delta f) \mathrm{kHz}$ \\
\hline Modeled NBI-bandwidth, $b f_{s} / N$ & $39(=\Delta f) \mathrm{kHz}$ \\
\hline Center-frequency, $f_{c}$ & $5.0 \mathrm{kHz}$ \\
\hline Center-frequency error, $\widehat{f}_{c}-f_{c}$ & $9.8(=0.25 \Delta f) \mathrm{kHz}$ \\
\hline
\end{tabular}

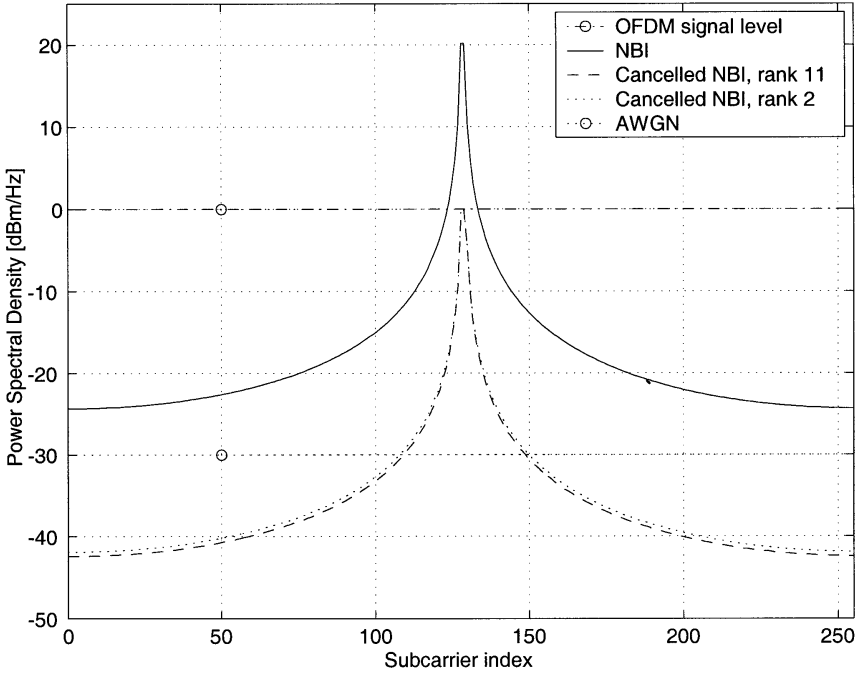

Fig. 17. Typical PSD levels without using any silent measurement tones.

the peak). Fig. 18 shows the SER with/without cancellation as a function of SNR, with $0 \mathrm{~dB}$ SIR. The rank-2 canceller shows a performance loss compared to the rank-3 and rank-11 cancellers for higher SNRs. The rank-3 canceller uses $3.1 \mathrm{mul}-$ tiplications per tone. A $(255,243)$ Reed-Solomon (RS) code and 16-QAM symbols was used and the average (received) signal power was $0 \mathrm{dBm} / \mathrm{Hz}$ on all subcarriers. The RS code was used without erasure decoding.

As a result of the cancellation, the SER has been lowered considerably despite the fact that no silent measurement tones were used. However, because of the latter there is an SER loss compared to using silent tones. For high SNR there is an SER floor due to the remaining residual NBI peak above the AWGN floor, see Fig. 17. However, the SER floor can be lowered by using erasures on the bad SNR tones (at the remaining NBI peak) in the RS decoding. Using silent tones for the canceller results in

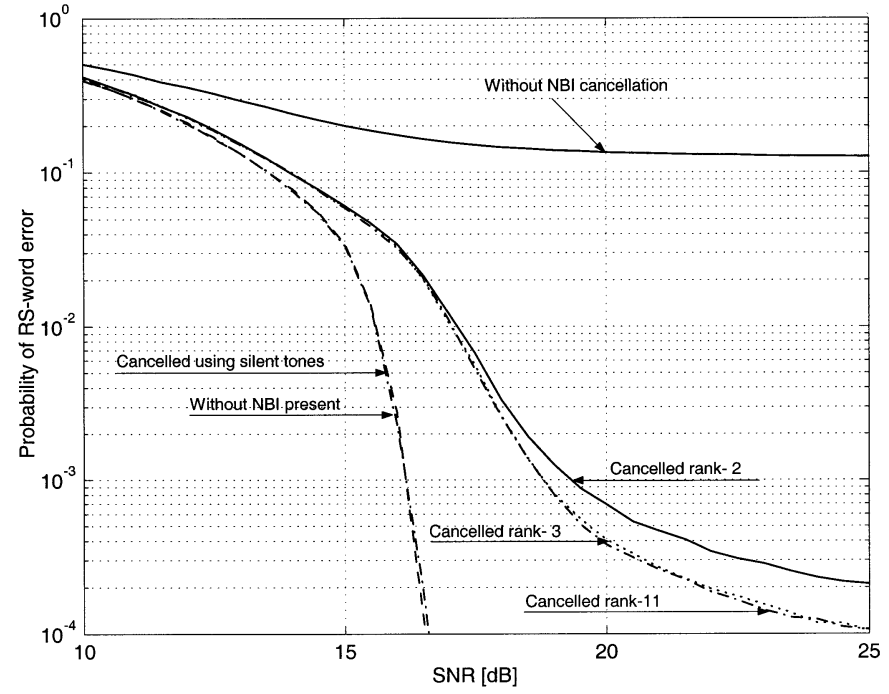

Fig. 18. Probability of RS(255,243)-word error versus SNR without using silent tones for canceller with rank 2, rank 3 , and rank 11 . SIR $=0 \mathrm{~dB}$. Word error rates for cancellation using silent tones, without NBI present and without NBI cancellation, is shown for comparision.

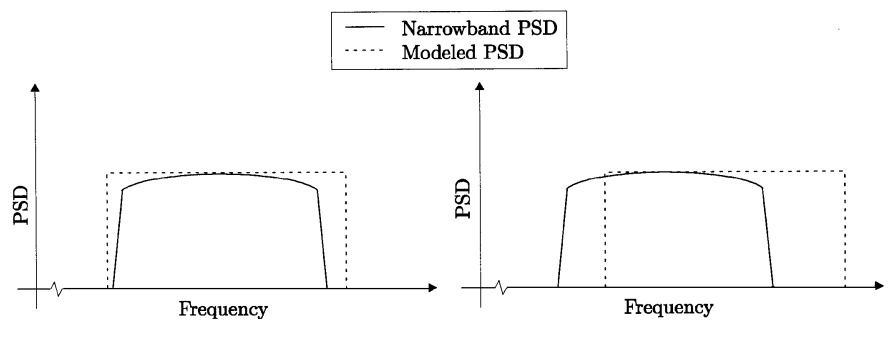

(a)

(b)

Fig. 19. (a) Good PSD model. (b) Poor PSD model.

nearly the same SER as if no NBI is present at all, the two corresponding curves are almost inseparable in Fig. 18.

\section{Conclusions Drawn From Examples}

The examples presented above show that it is possible to construct a robust canceller with only a rough knowledge of the NB signal's PSD. As long as the shape of the modeled PSD spans the true NB signal's PSD, as in Fig. 19, then the canceller will give good performance (residual PSD below background measurement noise floor) for various bandwidth and frequency errors.

However, the PSD model in Fig. 19(b) will produce poor results. For most effective rank reduction a flat shape of the modeled PSD is best. Then, the estimator energy is best concentrated 
to a few dimensions. NBI signals that have a flat PSD shape are also known to be the worst for block processed transmission systems [16].

A canceller that obeys this design rule will give almost as good performance as a canceller constructed with perfect knowledge of the NB signal's PSD and will be less sensitive to model errors. Using windowing reduces the number of subcarriers that need spectral cancellation at the price of a slightly reduced efficiency. Using measurement tones from more than one OFDM frame suppresses the interference even more but makes it more complex to cancel NB signals which may change its frequency location.

\section{SUMMARY}

An NBI canceller for OFDM systems has been derived. It can be used to suppress the spectral leakage that occurs when a strong NB signal resides in the same frequency band as the OFDM signal, which can be the case for example for DSL or in unlicensed frequency bands for radio transmission.

The canceller subtracts frequency-domain LMMSE estimates of the NBI from the received signal by measuring the interference on a few modulated or unmodulated OFDM tones. We use optimal rank reduction of the canceller and apply the time-bandwidth product on the NB signal to lower the run time computational complexity. This is especially valuable if many measurement tones are used and the NB signal bandwidth is small.

With cognizant design, the canceller can be made invariant to frequency shifts meaning that the estimator coefficients do not need to be updated if the NB signal suddenly changes its frequency location. This avoids extra computational complexity and makes it easier to cancel NB signals which may change their frequency location or to cancel several NB signals with different carrier frequencies.

Performance evaluations show that this approach leads to a robust canceller that performs good with only rough a priori information about the NB signal. It is possible to suppress the spectral leakage caused by an NB signal and reduce the average SNR loss from, e.g., 20 or $30 \mathrm{~dB}$ before cancellation to nearly $0 \mathrm{~dB}$ after cancellation.

Using a DMT-based VDSL system as a specific application scenario it is shown how the canceller can naturally operate in a standardized receiver structure to effectively suppress strong radio frequency interference caused by amateur radio. With a general OFDM-based receiver structure it also shown that it is still possible to get a quite dramatic SER improvement by using the NBI canceller and a standard block code even though no unmodulated OFDM subcarriers are available as measurement tones.

\section{ACKNOWLEDGMENT}

The authors would like to thank the anonymous reviewers for their helpful comments as well as the associate editor for his efforts and patience.

\section{REFERENCES}

[1] R. Wolters, "Characteristics of upstream channel noise in CATV networks," IEEE Trans. Broadcast., vol. 42, pp. 328-332, Dec. 1996.
[2] K. T. Foster, "The RFI environment for VDSL," presented at the IEEE Globecom '96, VDSL Workshop, London, U.K., Nov. 18, 1996.

[3] R. van Nee, G. Awater, M. Morikura, H. Takanashi, M. Webster, and K. W. Halford, "New high-rate wireless LAN standards," IEEE Commun Mag., vol. 37, pp. 82-88, Dec. 1999.

[4] "Transmission and multiplexing (TM) access transmission systems on metallic access cables; very high speed digital subscriber line (VDSL)—Part 2: Transceiver specification," ETSI STC TM6, Sophia Antipolis, France, Tech. Spec. TS 101 270-2 V1.1.1 (2001-02), Feb. 2001.

[5] L. L. Scharf, Statistical Signal Processing: Detection, Estimation, and Time Series Analysis. Reading, MA: Addison-Wesley, 1991.

[6] H. J. Landau and H. O. Pollak, "Prolate spheriodal wave functions, Fourier analysis and uncertainty-III: The dimension of the space of essentially time- and band-limited signals," Bell Syst. Tech. J., vol. 41, p. 1295, 1962.

[7] L. de Clercq, M. Peeters, S. Schelstraete, and T. Pollet, "Mitigation of radio interference in xDSL transmission," IEEE Commun. Mag., vol. 38, pp. 168-173, Mar. 2000.

[8] A. Peled and A. Ruiz, "Frequency domain data transmission using reduced computational complexity algorithms," in Proc. IEEE Int. Conf. Acoustics, Speech, and Signal Processing, Denver, CO, 1980, pp. 964-967.

[9] P. Spruyt, P. Reusens, and S. Braet, "Performance of improved DMT transceiver for VDSL," ANSI, Colorado Springs, CO, Tech. Rep. T1E1.4/96-104, Apr. 1996.

[10] J. A. C. Bingham, "RFI suppression in multicarrier transmission systems," in Proc. Globecom, vol. 2, London, U.K., Nov. 1996, pp. 1026-1030.

[11] B. Wiese and J. Bingham, "Digital radio frequency cancellation for DMT VDSL," ANSI, Sacramento, CA, Tech. Rep. T1E1.4/97-460, Dec. 1997.

[12] B.-J. Jeong and K.-H. Yoo, "Digital RFI canceller for DMT-based VDSL," Electron. Lett., vol. 34, pp. 1640-1641, Aug. 1998.

[13] F. Sjöberg, R. Nilsson, N. Grip, P. O. Börjesson, S. K. Wilson, and P. Ödling, "Digital RFI suppression in DMT-based VDSL systems," in Proc. Int. Conf. Telecommunications (ICT), vol. 2, Chalkidiki, Greece, June 1998, pp. 189-193.

[14] B. Picinbono, "Second-order complex random vectors and normal distributions," IEEE Trans. Signal Processing, vol. 44, pp. 2637-2640, Oct. 1996.

[15] P. J. Schreier and L. L. Scharf, "Second-order analysis of improper complex random vectors and processes," IEEE Trans. Signal Processing, vol. 51, pp. 714-725, Mar. 2003.

[16] A.-D. Naofal and S. H. Diggavi, "Maximum throughput loss of noisy ISI channels due to narrow-band interference," IEEE Commun. Lett., vol. 5, pp. 233-235, June 2001.

[17] B. Picinbono and P. Chevalier, "Widely linear estimation with complex data," IEEE Trans. Signal Processing, vol. 43, pp. 2030-2033, Aug. 1995.

[18] O. Edfors, M. Sandell, J.-J. van de Beek, S. K. Wilson, and P. O. Börjesson, "OFDM channel estimation by singular value decomposition," IEEE Trans. Commun., vol. 46, pp. 931-939, July 1998.

[19] "Very-high-speed digital subscriber lines-System requirements," ANSI, Plano, TX, Tech. Rep. T1E1.4/98-043R8, Nov. 1998.

[20] G. Forney and M. Eyuboglu, "Combined equalization and coding using precoding," IEEE Commun. Mag., vol. 29, pp. 25-34, Dec. 1991.

[21] J. Proakis, Digital Communications, 3rd ed. Englewood Cliffs, NJ: Prentice-Hall, 1995.

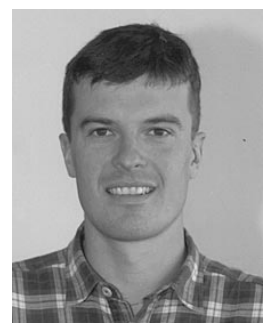

Rickard Nilsson (S'96-A'03) was born in Umeå, Sweden, in 1971. He received the M.S.E.E. degree in 1996, the licentiate of engineering in 1999, and the Ph.D. degree in signal processing in 2002, all from Luleå University of Technology, Luleå, Sweden.

Between 1997-2002, he worked cooperatively with Telia Research AB, Sweden, by contributing to the development and standardization of the DMT-Zipper method for VDSL. During the fall semester in 1999, he was a Guest Researcher at Stanford University, CA. In May 2002, he moved from the arctic north in Luleå to historic Vienna and joined the Telecommunications Research Center Vienna (ftw.), Vienna, Austria. His research interests include multicarrier modulation, statistical signal processing, low-complexity algorithms, interference suppression, synchronization. 


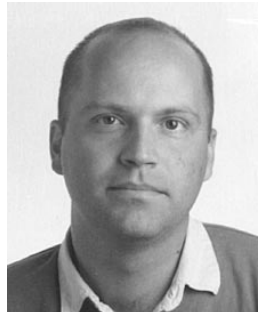

Frank Sjöberg (S'95-M'00) was born in Umeå, Sweden, in 1970. He received the M.S. degree in computer science and the Ph.D. degree in signal processing from Luleå University of Technology, Luleå, Sweden, in 1995 and 2000, respectively.

Between 2000-2002 he was with Telia Research AB. He is currently an Assistant Professor in the Division of Signal Processing, Luleå University of Technology. His primary research interests are statistical signal processing and digital communication, with emphasis on wireline systems.

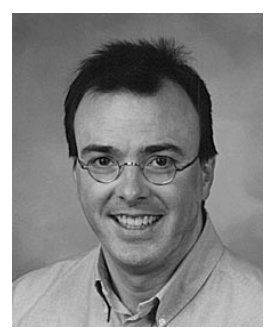

James P. LeBlanc (S'91-M'95-SM'98) was born in Kenmore, NY, in 1961. He received the B.S. degree from Rensselaer Polytechnic Institute, Troy, NY, in 1983, and the Ph.D. degree from Cornell University, Ithaca, NY, in 1995, both in electrical engineering.

He worked in industry for several years and was a member of the academic staff of New Mexico State University. In 2000, he joined Luleå University of Technology, Luleå, Sweden. His interests are statistical signal processing, blind equalization/identification, and systems theory. 